\title{
Transcriptome analysis reveals a positive effect of brassinosteroids on the photosynthetic capacity of wucai under low temperature
}

Mengru Zhao ${ }^{1,2+}$, Lingyun Yuan ${ }^{1,2,3 \dagger}$ D, Jie Wang ${ }^{1,2}$, Shilei Xie ${ }^{1,2}$, Yushan Zheng ${ }^{1,2}$, Libing Nie ${ }^{1,2}$, Shidong Zhu ${ }^{1,2,3}$, Jinfeng Hou ${ }^{1,2,3}$, Guohu Chen ${ }^{1,2,3}$ and Chenggang Wang ${ }^{1,2,3^{*}}$ (i)

\begin{abstract}
Background: Brassinosteroids (BRs) have a positive effect on many processes during plant growth and development, and in response to various abiotic stressors. Low-temperature (LT) stress constricts the geographic distribution, growth, and development of wucai (Brassica campestris L. ssp. chinensis var. rosularis Tsen). However, there is little information on the global gene expression of BRs under LT stress in wucai. In this study, the molecular roles of 24-epibrassinolide (EBR) after exogenously application, were explored by RNA sequencing under LT conditions.

Results: According to the Gene Ontology (GO) term and Kyoto Encyclopedia of Genes and Genomes (KEGG) pathway analyses, photosynthesis was significantly enriched after spraying EBR under LT. The transcripts encoding the photosystem II (PSII) oxygen-evolving enhancer protein, photosystem I (PSI) subunit, light-harvesting chlorophyll protein complexes I and II, and ferredoxin were up-regulated after the application of EBR. Transcripts encoding several key enzymes involved in chlorophyll biosynthesis were also up-regulated, accompanied by significant differences in the contents of 5-aminolevulinic acid (ALA), porphobilinogen (PBG), protoporphyrin IX (Proto IX), Mg-protoporphyrin IX (Mg-proto IX), protochlorophyllide (Pchl), and photosynthetic pigments. Notably, transcriptional and physiological analyses revealed that under LT stress, plant responses to EBR involved a major reorientation of photosynthesis, as well as porphyrin and chlorophyll metabolism.

Conclusion: This study explored the role of EBR as an LT stress tolerance mechanism in wucai. At the transcription level, LT tolerance manifests as an enhancement of photosynthesis, and the amelioration of porphyrin and chlorophyll metabolism.
\end{abstract}

Keywords: RNA-Seq, Low-temperature stress, Photosynthesis, Porphyrin and chlorophyll metabolism

\footnotetext{
* Correspondence: cgwang@ahau.edu.cn

${ }^{\dagger}$ Mengru Zhao and Lingyun Yuan contributed equally to this work.

'College of Horticulture, Vegetable Genetics and Breeding Laboratory, Anhui

Agricultural University, 130 West Changjiang Road, Hefei 230036, Anhui,

China

${ }^{2}$ Provincial Engineering Laboratory for Horticultural Crop Breeding of Anhui,

130 West of Changjiang Road, Hefei 230036, Anhui, China

Full list of author information is available at the end of the article
}

(c) The Author(s). 2019 Open Access This article is distributed under the terms of the Creative Commons Attribution 4.0 International License (http://creativecommons.org/licenses/by/4.0/), which permits unrestricted use, distribution, and reproduction in any medium, provided you give appropriate credit to the original author(s) and the source, provide a link to the Creative Commons license, and indicate if changes were made. The Creative Commons Public Domain Dedication waiver (http://creativecommons.org/publicdomain/zero/1.0/) applies to the data made available in this article, unless otherwise stated. 


\section{Background}

Wucai (Brassica campestris L. ssp. chinensis var. rosularis Tsen) is a variant of non-heading Chinese cabbage (Brassica campestris L.), a crucial species in the Brassicaceae family [1]. As an important autumn and winter vegetable crop, wucai is cultured throughout most parts of China, especially in the Yangtze-Huaihe River Basin. With a beautiful shape and high levels of 70 vitamins and minerals, wucai is becoming an increasingly popular crop in other countries.

Brassinosteroids (BRs) were isolated from Brassica pollen $\sim 40$ years ago, and they are a type of plant steroid hormone. 24-epibrassinolide (EBR), one of the BRs, is biologically-active brassinolides hormones used in physiological and molecular studies [2]. Moreover, previous studies found that BRs regulate a variety of physiological and developmental processes, including plant stress tolerance, leaf elongation, and flower development, which up-regulate large genes involved in cell division and differentiation, and exhibit control over all developmental processes [3]. BRs are known to regulate photosynthesis under normal as well as in abnormal conditions [4, 5]. The studies of Choudhary et al. [6] and Yuan et al. [7] showed that foliar application of EBR can increase plant chlorophyll (Chl) content and up-regulate the expression levels of various oxidative stress marker genes, thereby improving photosynthetic capacity to enhance the tolerance of Arabidopsis and Cucumis sativus L. responding to different stresses.

Low-temperature (LT) stress is harmful to numerous plant physiological processes and is the main abiotic stressor that limits plant growth and development. For example, LT stress can stunt plant growth, disrupt photosynthesis, and reduce chlorophyll content, resulting in significant yield and economic losses. Recently, our team reported that the differentially expressed

Cont, $\mathrm{LT}, \mathrm{LT}+0.05 \mu \mathrm{M}, \mathrm{LT}+0.08 \mu \mathrm{M}, \mathrm{LT}+0.1 \mu \mathrm{M}, \mathrm{LT}+0.12 \mu \mathrm{M}, \mathrm{LT}+0.15 \mu \mathrm{M}$

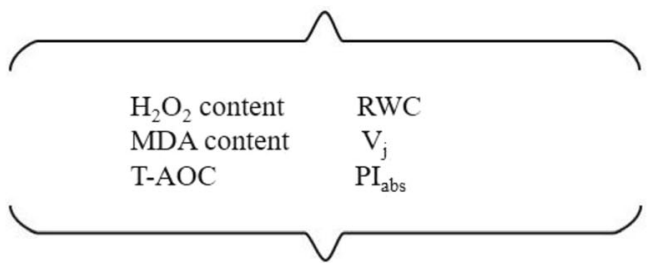

Identification of EBR concentration $(0.1 \mu \mathrm{M})$
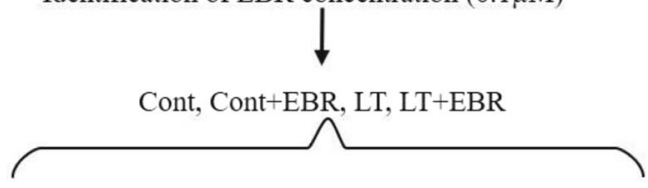

Total soluble sugar content

MDA content SLA

MSI

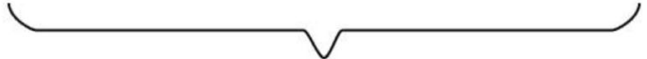

Identification of treatment groups for RNA-seq (LT, LT+EBR)

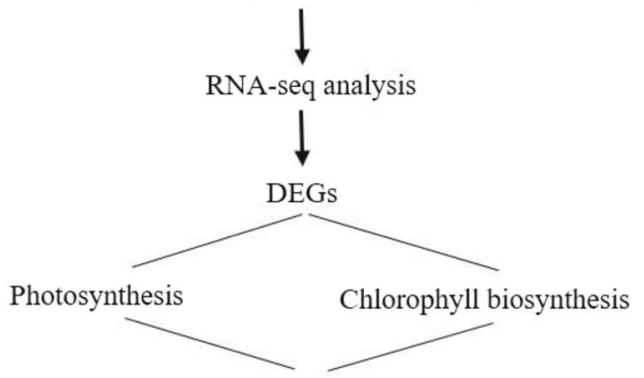

Brassinosteroids plays a positive role in the photosynthetic capacity of wucai under low temperature

Fig. 1 Experimental design ideas 
proteins in wucai (Brassica campestris L.) produced by temperature stress were mainly enriched in porphyrin and chlorophyll metabolism, carbon metabolism, carbon fixation in photosynthetic organisms, and so on [8]. Previous research demonstrated that the exogenous application of BRs significantly improved resistance to LT stress by regulating the morphological, physiological, and biochemical characteristics of pepper (Capsicum annuum L.) $[9,10]$, tomato (Solanum lycopersicum Mill.) [11], and cucumber (Cucumis sativus L.) [12]. Transcriptome analysis indicated that the DEGs of Winter Rapeseed (Brassica rapa L.) under cold stress were significantly enriched in plant hormone signal transduction, starch and sucrose metabolism, and photosynthesis [13]. Jaglo illustrated that the freezing tolerance of $B$. napus can be enhanced through CBF-mediated "regulon engineering" [14]. The results of these studies indicate that exogenous applications of BRs reduce the adverse effects of LT stress by increasing chlorophyll content, maintaining photosynthesis, and activating gene expression and signal transduction pathways $[15,16]$.

RNA-Seq technology is a high-throughput transcriptome analysis platform that can expeditiously, accurately, and economically explore instantaneous gene expression of multiple, diverse, and even non-model species [17]. In this study, RNA-Seq analysis was conducted in order to explore how gene expression correlates with LT stress and the signaling pathways regulated by 24-epibrassinolide (EBR). A physiological analysis of chlorophyll biosynthesis and photosystem activity was also conducted in order to identify a key pathway for transcriptional regulation (Fig. 1). The goals of this study were to: (1) elucidate the transcriptomic response to EBR and LT stress; (2) reveal the genes and pathways that correlate with EBR-induced cold tolerance; and, (3) determine the interactive influences of EBR and LT stress on leaf photosystem activity and chlorophyll biosynthesis in wucai. The findings of this work will elucidate the underlying mechanisms of this plant growth regulator regulation and the response mechanisms of no-heading cabbage species to LT stress.

\section{Results}

\section{Physiological parameter effects of EBR}

The physiological parameters reflecting plant cold injury (e.g., specific leaf area (SLA), membrane stability index (MSI), total soluble sugar content, and malondialdehyde (MDA)) were not significantly different between spraying with EBR (Cont+EBR) and the control (Cont). When subjected to LT stress, spraying EBR (LT + EBR) led to significant differences in SLA (Fig. 2a), MSI (Fig. 2b), total sugar content (Fig. 2c), and MDA (Fig. 2d) content compared to LT plants. Temperature had a significant effect and all 4 of the parameters were significantly different between LT and Cont. There were no significant differences in SLA, total soluble sugar content, and MDA between LT + EBR and Cont (Fig. 2).

The largest change was in the total soluble sugar content among the 4 indicators. Compared to Cont, the

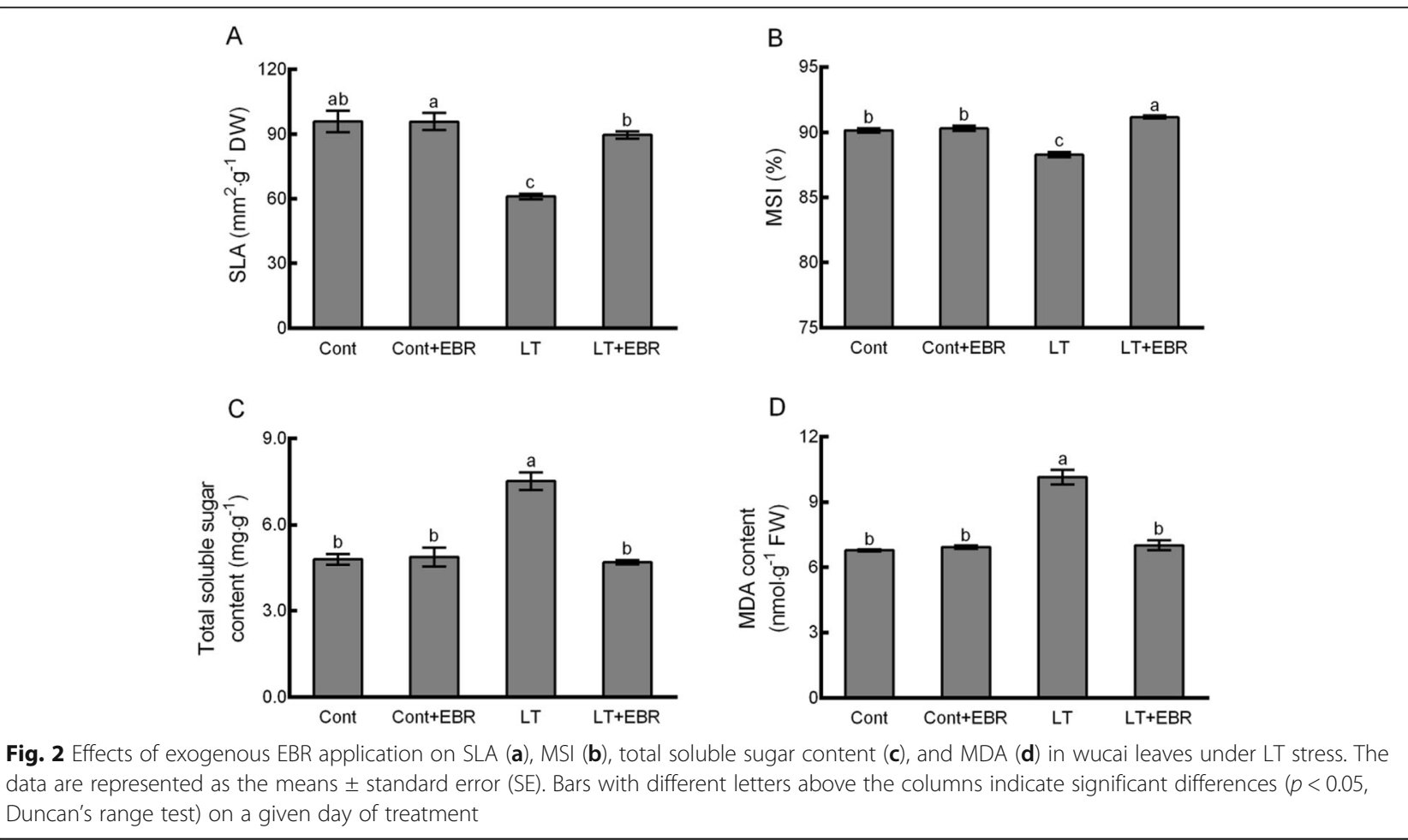


total soluble sugar content increased by $56.87 \%$ in LT plants (Fig. 2c). Furthermore, the total soluble sugar content of LT plants significantly decreased after spraying EBR under LT stress (37.59\%, Fig. 2c). The MDA content followed a similar trend. Compared to Cont, MDA content significantly increased by $49.81 \%$ in LT plants, and significantly decreased by $30.95 \%$ in LT + EBR (Fig. 2d). For the LT treatments specifically, SLA was depressed by $36.33 \%$. Compared to LT, SLA significantly increased by $46.80 \%$ in LT + EBR plants (Fig. 2a). Compared to Cont, the MSI content of LT was significantly reduced by $2.07 \%$. In contrast, MSI significantly increased by $1.14 \%$ after EBR treatment (Fig. 2b). Compared to LT, SLA significantly increased by $3.27 \%$ in LT + EBR (Fig. 2a).

\section{Mapping and quantitative assessment of Illumina sequences}

Two libraries were constructed from the LT and LT + EBR treatment groups for analysis by RNA-Seq. A total of 53.51 million (LT) and 50.83 million (LT + EBR) reads were generated. After removing the adapter, poly- $\mathrm{N}$, low-quality, and empty reads, $>6$ billion clean bases were obtained with a Q30 percentage $>94 \%$, and a GC percentage between 47.447.7\% (Additional file 5: Table S1). The quality control (fastqc v0.11.5, Illumina) and reads mapping (fastqc v0.11.5, Illumina) were shown in Additional file 1: Figure S1 and Additional file 2: Figure S2.

Each library of clean reads was aligned to the Brassica rapa (B. rapa) reference genome [18]. The proportion of clean reads in the 2 wucai transcriptome libraries that mapped to the $B$. rapa reference genome ranged from $86.67-87.56 \%$ (Additional file 6: Table S2). A total of 30,843 genes were confirmed from the mapped libraries. All of the RNA- sequence data in this article have been deposited in the NCBISRA database and are accessible in SRP200451 (https://www. ncbi.nlm.nih.gov/sra/SRP200451).

\section{Transcriptome profiles}

The 30,843 genes from the mapped libraries were normalized using the reads per kilo bases per million reads (RPKMs) method [19]. A total of 2443 differentially expressed genes (DEGs) were identified between LT and LT + EBR (Fig. 3a, b). Hierarchical clustering of all of the DEGs was conducted to observe the gene expression patterns and was evaluated by the $\log _{10}$ RPKMs of the 2 groups (Fig. 3c). Compared to LT, LT + EBR contained 1581 up-regulated and 862 downregulated genes. These results suggest that EBR had a marked effect on the transcription of a subset of genes in response to LT stress.

\section{GO and KEGG analyses of the EBR-induced DEGs}

In order to explore the function of the EBR-induced DEGs under LT stress, the DEGs were used in the Gene
Ontology (GO) enrichment analysis. Results uncovered the biological process, molecular function, and cellular component categories for the 2443 DEGs of the 2 groups (Fig. 4a). The transcriptome analysis sifted GO terms with the number of DEGs $>2$ in the 3 classifications, according to the corresponding - $\log _{10}$ Pvalue of each term; 10 terms were sorted from large to small. In the biological process category, cellular response to far red light (GO:0071490) was the most abundant, followed by cellular response to red light (GO:0071491), indicating the positive regulation of reactive oxygen species biosynthetic processes (GO:1903428), such as photosynthesis and light harvesting in photosystem I (GO: 0009768). Similarly, in the cellular component category, photosystem I (GO:0009522) was the most abundant, followed by plastoglobule (GO:0010287), chloroplast thylakoid membrane (GO:0009535), and photosystem II (GO:0009523). In the molecular function category, oxygen-evolving activity (GO:0010242) was the most abundant, followed by chlorophyll binding (GO: 0016168), pigment binding (GO:0031409), transcription factor activity, and sequence-specific DNA binding (GO: 0003700). Besides, the expression patterns of the cold acclimation, cold-induced and BR-responsive genes were listed in Additional file 7: Table S3 and Additional file 8: Table S4.

Among the DEGs, there were 7 pathways with Kyoto Encyclopedia of Genes and Genomes (KEGG) annotations that were significantly affected: (1) photosynthesisantenna proteins, (2) photosynthesis, (3) plant hormone signal transduction, (4) flavonoid biosynthesis, (5) pentose and glucuronate interconversions, (6) quorum sensing, and (7) phenylpropanoid biosynthesis $(p<0.001$; Fig. $4 \mathrm{~b})$. Higher enrichment scores and more genes were observed in photosynthesis-antenna proteins (24 genes) and photosynthesis (24 genes).

\section{Quantitative real-time PCR (qRT-PCR) analysis}

In order to validate the DEG data from RNA-Seq, 20 DEGs were randomly selected for quantitative real-time (qRT)-PCR assays in EBR-mediated LT stress (Additional file 3: Figure S3). Results indicated a strong positive correlation with the RNA-Seq data $\left(R^{2}=0.917\right)$, thereby validating the RNA-Seq data (Fig. 5; Additional file 9: Table S5).

\section{Effects of EBR on Porphyrin and chlorophyll metabolism analysis}

From the KEGG pathway analysis, results revealed that an exogenous EBR pretreatment can up-regulate the expression of several genes in porphyrin and chlorophyll metabolic pathways under LT conditions compared to Cont (Fig. 6). These up-regulated genes encode enzymes that cover almost the whole process of 5 -aminolevulinic 

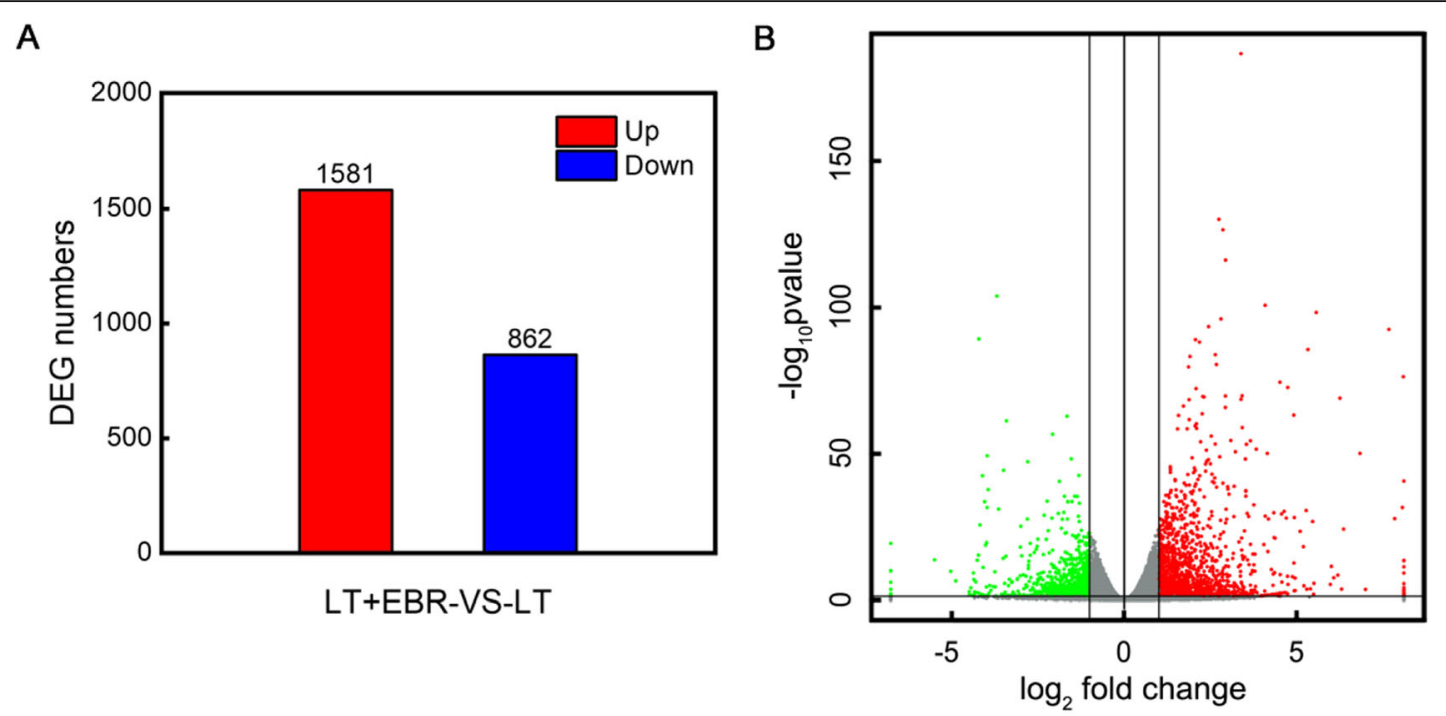

C

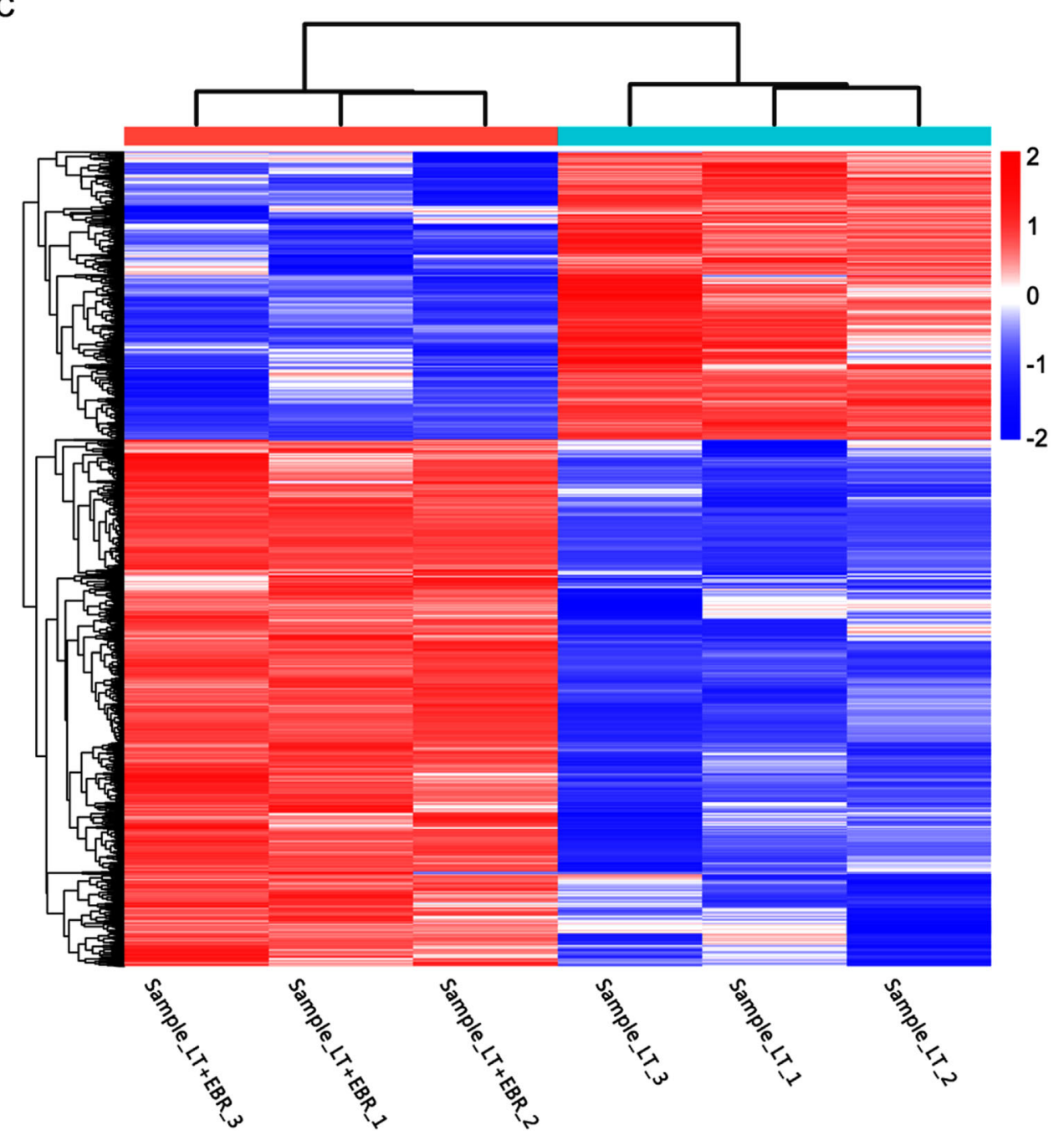

Fig. 3 (See legend on next page.) 
(See figure on previous page.)

Fig. 3 Transcriptome analysis of the DEGs in the $L T$ and $L T+E B R$ treatments of wucai leaves. a Comparisons of DEGs in the $L T$ and $L T+E B R$ treatments. $\mathbf{b}$ Volcano plot showing the DEGs between two different libraries. The threshold $q<0.05$ was used to determine the significance of DEGs. Red and green dots represent up- and down-regulated genes, respectively, and gray dots indicate transcripts that did not change significantly in the LT + EBR library compared to LT. c Hierarchical clustering of all of the DEGs was based on the $\log _{10} R P K M$ values. The color spectrum from blue to red represents the gene expression intensity from low to high, respectively

acid (ALA) and chlorophyll biosynthesis, including uroporphyrinogen decarboxylase (EC:4.1.1.37), coproporphyrinogen III oxidase (EC:1.3.3.3), magnesiumprotoporphyrin O-methyltransferase (EC:2.1.1.11), and protochlorophyllide reductase (EC:1.3.1.33). The downregulated gene encodes chlorophyllase (EC:3.1.1.14).

In order to determine whether exogenous EBR treatment influenced the photosynthetic pigment contents and metabolite levels involved in porphyrin and chlorophyll metabolism, the contents of chlorophyll $a(\mathrm{Chl} a)$, chlorophyll $b(\mathrm{Chl} b)$, total chlorophyll (Total Chl) content, the Chl $a / b$ ratio, ALA, porphobilinogen (PBG), protoporphyrin IX (Proto IX), Mg-protoporphyrin IX (Mg-Proto IX), and protochlorophyllide (Pchl) were assessed. No significant differences were detected between Cont and Cont+EBR (Figs. 7 and 8). Significant increases in Chl $a$, Chl $b$, Total Chl content, ALA, and PBG were detected in LT + EBR plants after $5 \mathrm{~d}$ compared to LT, with increases of 24.44, 18.41, 22.56, 59.01, and $52.06 \%$, respectively. In contrast, the contents of Proto IX, Mg-Proto IX, and Pchl significantly declined by $25.45,24.97$, and $25.78 \%$, respectively, when LT + EBR was compared to LT. Additionally, LT resulted in significant declines in Chl $a$, Total Chl content, ALA, and PBG by $11.41,10.47,10.04$, and $33.89 \%$, respectively, when LT was compared to Cont. Conversely, significant increases by $34.25,33.22$, and $48.58 \%$ were noted in the Proto IX, Mg-Proto IX, and Pchl contents, respectively, when LT was compared to Cont. There were no significant differences detected between LT + EBR and Cont/ Cont+EBR, except inthe Chl $a$ and ALA contents.

\section{Effect of EBR on photosynthesis}

In LT + EBR plants, the genes involved in photosynthesis were primarily up-regulated (Fig. 9), with a focus on PSII, PSI, and the light-harvesting chlorophyll protein complex (LHC) (Fig. 10a, b). In PSII, the DEGs included oxygen-evolving enhancer protein 1 (Psb O), oxygenevolving enhancer protein 2 (Psb P), oxygen-evolving enhancer protein 3 (Psb Q), $10 \mathrm{kDa}$ protein (Psb R), and Psb27 protein (Psb 27). In PSI, the DEGs included subunit II (Psa D), subunit III (Psa F), subunit V (Psa G), subunit VI (Psa $\mathrm{H})$, subunit X (Psa K), and subunits Psa $\mathrm{N}$ and Psa $\mathrm{O}$. The chlorophyll a/b-binding protein was also up-regulated, which is well-known as an important component of the light-harvesting chlorophyll protein complexes I and II [20]. In total, 23 genes encoding the chlorophyll a/b-binding protein were enhanced by EBR application (Fig. 11, Additional file 10: Table S6, Additional file 11: Table S7).

The Chl $a$ fluorescence transient reflects the effect of PSII afterquantitatively analyzing changes in the OJIP curve (Fig. 12). The radar maps show the changes in the receptor side and reflection center of PSII (Fig. 13). LT was associated with significantly higher $\mathrm{V}_{\mathrm{j}}, \mathrm{DI}_{0} / \mathrm{RC}$, and minimum fluorescence $\left(F_{0}\right)$ than the other 3 groups. In contrast, $\mathrm{Fv} / \mathrm{F}_{0}, \phi \mathrm{Eo}, \psi_{\mathrm{o}}$, and $\mathrm{PI}_{\mathrm{abs}}$ in LT plants were significantly lower compared to the other 3 groups (Fig. 14, Additional file 12: Table S8). For all of the parameters, there were no significant differences detected between Cont and Cont+EBR. There were also no significant differences detected in $\mathrm{RC} / \mathrm{CS}_{0}, \phi \mathrm{Do}$, and $\phi$ Po between LT and $\mathrm{LT}+\mathrm{EBR}$. LT was associated with significantly higher $\mathrm{V}_{\mathrm{j}}, \mathrm{DI}_{0} / \mathrm{RC}$, and $\mathrm{F}_{0}$ compared to Cont, Cont+EBR, and $\mathrm{LT}+\mathrm{EBR}$. In contrast, $\mathrm{Fv} / \mathrm{F}_{0}, \phi E o, \psi_{\mathrm{o}}$, and $\mathrm{PI}_{\mathrm{abs}}$ in LT plants were significantly lower compared to the other 3 groups. There were no significant differences detected between Cont and Cont+EBR for all of the parameters. There were also no significant differences detected in $\mathrm{RC} / \mathrm{CS}_{0}, \phi \mathrm{Do}$, and $\phi \mathrm{Po}$ between LT and LT + EBR; however, a $3.18 \%$ decrease in $\phi$ Po was observed when LT was compared to Cont. As for $\mathrm{RC} / \mathrm{CS}_{0}$ and $\phi \mathrm{Do}$, there were significant increases of 28.37 and $16.58 \%$, respectively, in $\mathrm{LT}$ compared to Cont. In $\mathrm{LT}+\mathrm{EBR}$, the $\mathrm{V}_{\mathrm{j}}, \mathrm{DI}_{0} /$ $\mathrm{RC}, \mathrm{F}_{0}, \mathrm{Fv} / \mathrm{F}_{0}, \phi E \mathrm{E}, \psi_{\mathrm{o}}$, and $\mathrm{PI}_{\mathrm{abs}}$ concentrations significantly changed compared to LT. Among the abovementioned factors, $\mathrm{V}_{\mathrm{j}}, \mathrm{DI}_{0} / \mathrm{RC}$, and $\mathrm{F}_{0}$ were significantly reduced by $13.80,6.24$, and $7.00 \%$, respectively. Conversely, $\mathrm{Fv} / \mathrm{F}_{0}, \phi E o, \psi_{\mathrm{o}}$, and $\mathrm{PI}_{\mathrm{abs}}$ increased significantly by $3.42,9.69,9.12$, and $34.38 \%$, respectively, in LT + EBR compared to LT (Fig. 14).

\section{Discussion}

BRs are a series of naturally present plant steroids associated with several critical physiological and cellular plant processes when experiencing a variety of environmental stressors [21]. BRs are a category of steroidal growth regulators that promote stress tolerance and crop yield [22, 23]. Although previous studies on BRs have demonstrated that the functions of exogenous BRs can mitigate the damage induced by abiotic stress [24, 25], the associated regulatory mechanisms, especially the underlying molecular mechanisms, have received little attention. In this study, through physiological and 


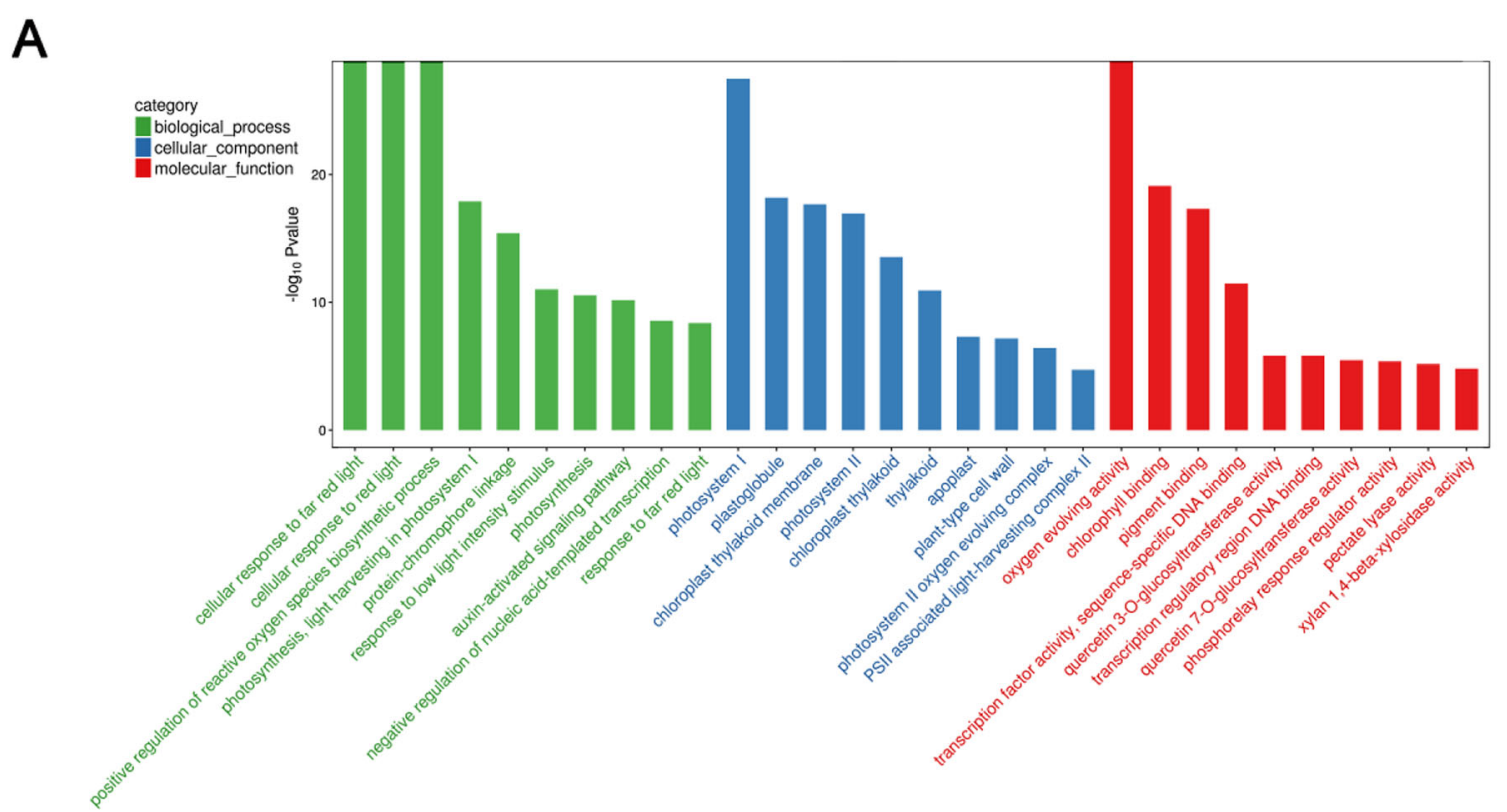

B

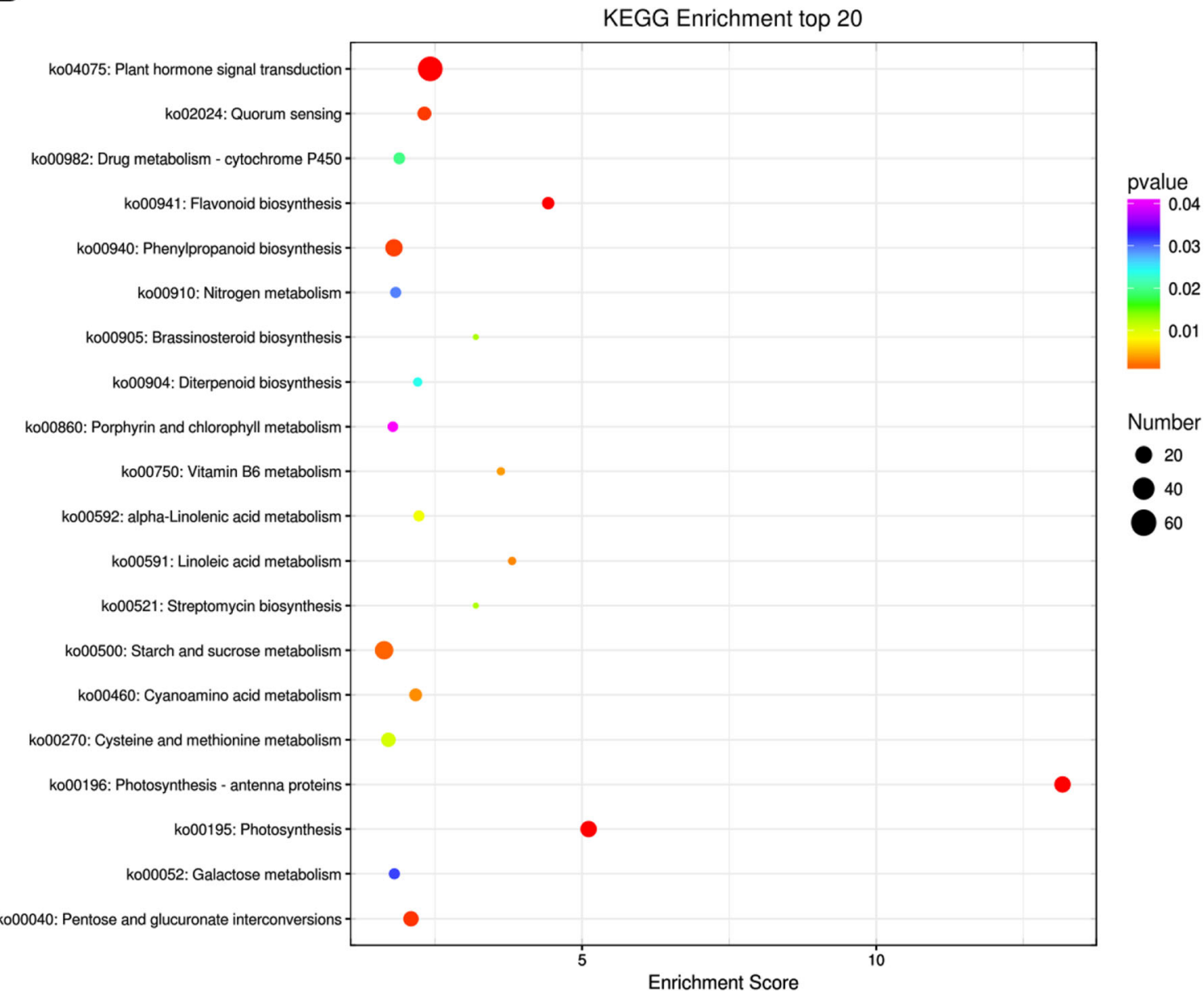

Fig. $4 \mathrm{GO}$ and KEGG pathway enrichment analyses of the DEGs in the LT and LT + EBR treatments of wucai leaves. a GO enrichment analysis with the 30 most enriched GO terms in the 3 categories shown. b KEGG enrichment analysis with the 20 most enriched KEGG terms shown. High and low $p$-values are represented by blue and red, respectively 


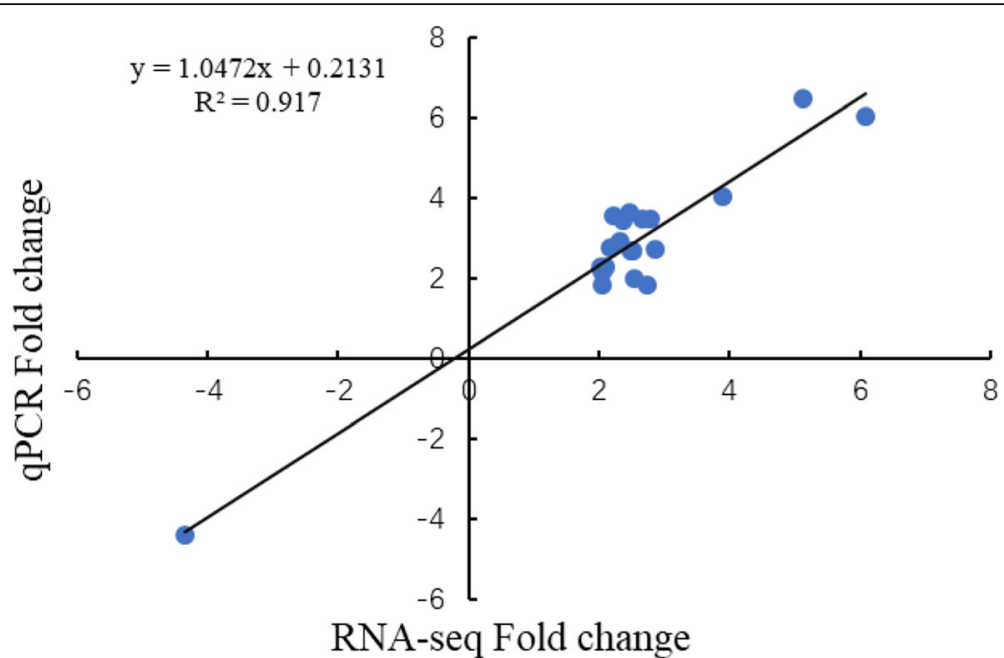

Fig. 5 Correlation of RNA-Seq (x-axis) qRT-PCR data (y-axis). The assay was carried out for 20 randomly selected DEGs. GO and KEGG enrichment analyses

biochemical analyses, it was demonstrated that an EBR pretreatment improved the cold tolerance of wucai. Then, in order to focus on the EBR-mediated variety in gene expression, RNA-Seq was used to investigate the part of EBR that responded to LT at the global transcriptome level. To the best of our knowledge, this study is the first demonstration of the molecular mechanisms of wucai responses to EBR treatments under LT. In order to determine the effect of EBR on LT tolerance, the discussion is focused on contrasting the LT and $\mathrm{LT}+\mathrm{EBR}$ treatment groups. Based on the GO and KEGG analyses, DEGs correlated with the application of

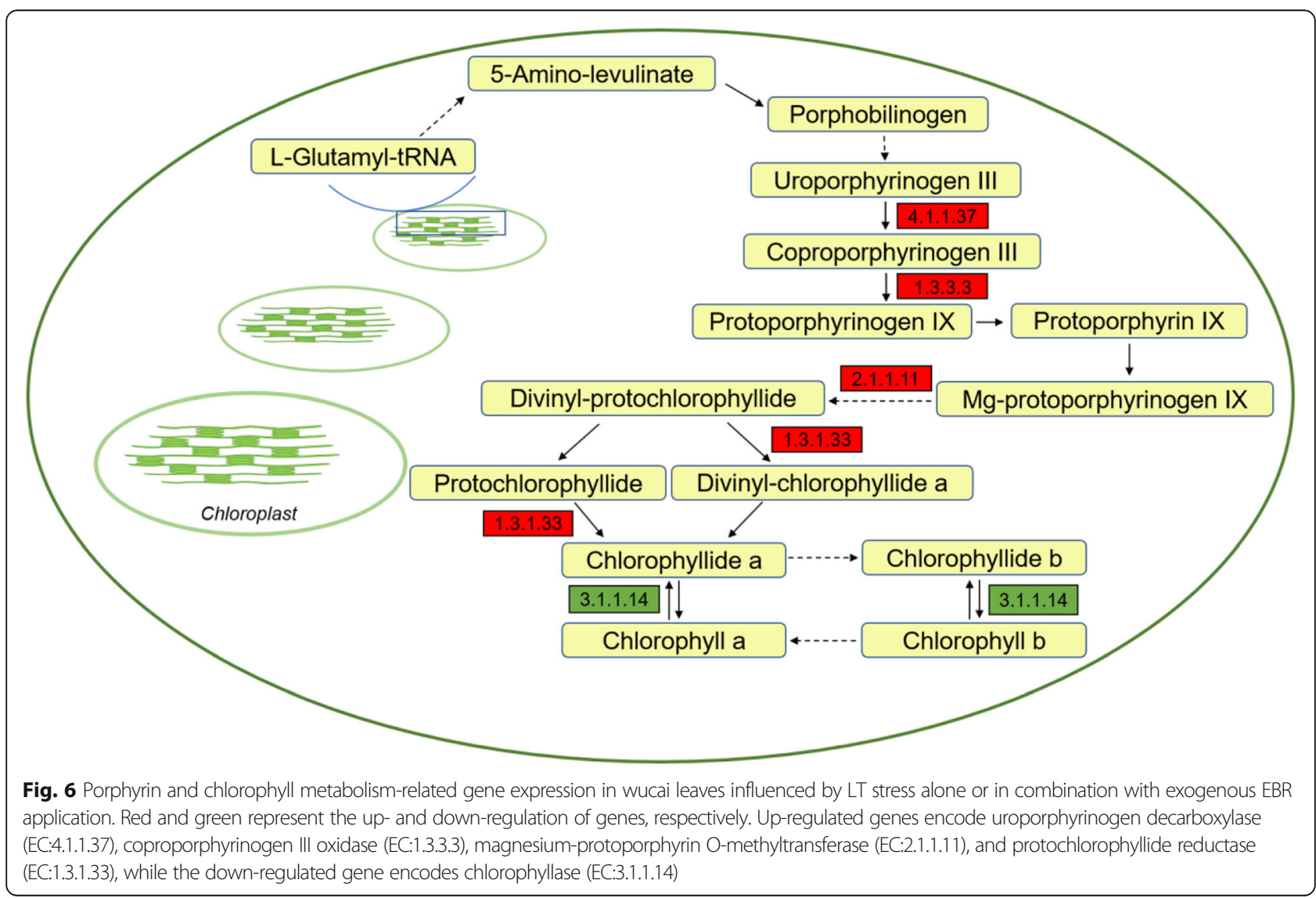



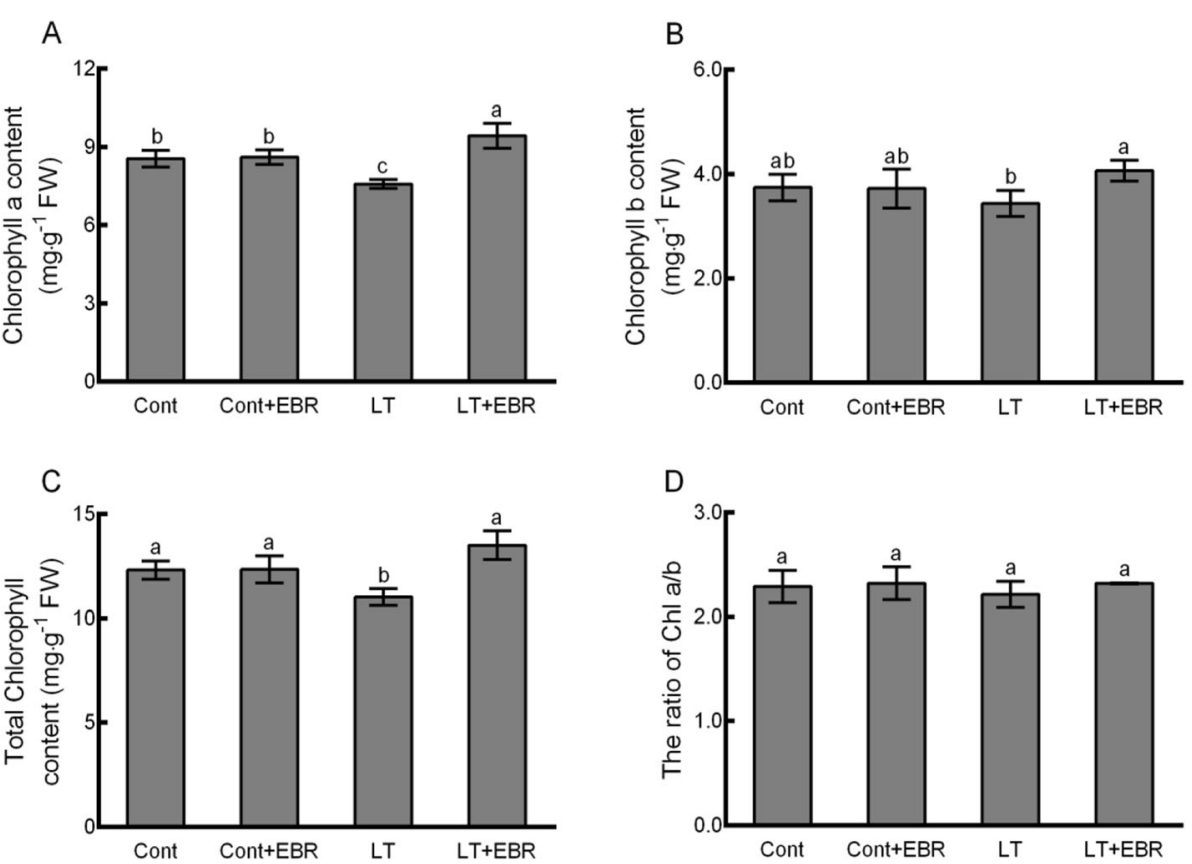

Fig. 7 Effects of exogenous EBR application on photosynthetic pigment contents in wucai leaves under LT stress. (A-C) Quantification of the Chl a content $(\mathbf{a})$, Chl $b$ content $(\mathbf{b})$, Total $\mathrm{Chl}$ content $(\mathbf{c})$, and the $\mathrm{Chl} a / b$ ratio calculated from $\mathrm{A}$ and $\mathrm{B}(\mathbf{d})$. The data are presented as the mean $\pm \mathrm{SE}$. Bars with different letters above the columns indicate significant differences $(p<0.05$, Duncan's range test) on a given day of treatment

exogenous EBR under LT were notably enriched in genes associated with photosynthesis and chlorophyll biosynthesis.

\section{Physiological parameters verify the role of exogenous EBR}

Variations in leaf structure patterns represent the adaptation of many species [26]. Higher SLA helps with higher potential evaporation requirements and wider foliar display, capturing more light for use in constant biomass investment [27]. MDA content is a main indicator for evaluating the degree of membrane oxidation. Low MDA content leads to a higher tolerance of LT stress. Soluble sugars play a prominent, central role in plant structure and metabolism at the cellular and whole organism levels [28]. MSI can be used to assess the integrity of the plasma membrane [29]; the higher the MSI value, the more complete the plasma membrane. In this study, the results of SLA, MSI, total soluble sugar content, and MDA indicate that EBR can significantly alleviate injury to LT. In order to explore the underlying molecular mechanisms, transcriptome sequencing was performed on $\mathrm{LT}$ and $\mathrm{LT}+\mathrm{EBR}$ plants.

\section{Exogenous EBR improves chlorophyll biosynthesis by promoting the conversion of intermediates}

Chlorophyll is an important component in photosynthesis, as it plays a role in the transmission, distribution, and transformation of light energy [20]. Chlorophyll content is closely related to photosynthetic rate and organic matter accumulation [30]. Some studies have shown that exogenous application of EBR accelerates the increase of chlorophyll content in plants under stress [31-33]. Chlorophyll metabolism is an intricate pathway that involves the biosynthesis and degradation of chlorophyll. Chlorophyll biosynthesis is catalyzed by a diverse set of enzymes and can be restrained by abating the activity of all of these enzymes [34]. The results of this study indicate that exogenous EBR treatment can increase the content of photosynthetic pigments under LT stress and promote a spectrum of enzymatic reactions in chlorophyll biosynthesis.

ALA is the first precursor of chlorophyll biosynthesis. Therefore, ALA biosynthesis has a considerable effect on the rate of chlorophyll biosynthesis [20]. In this study, a significant increase in endogenous ALA was detected in LT + EBR plants compared to LT. Additionally, the conversion of ALA to chlorophyll includes many intermediates such as PBG, Proto IX, Mg-Proto IX, and Pchl. EBR application caused significant increases in the ALA and PBG contents, and significant decreases in Proto IX, Mg-Proto IX, and Pchl compared to LT. Four transcripts encoding 4 of the key enzymes involved in catalyzing the synthesis of these products were all up-regulated by exogenous EBR application. CHLM encodes a magnesium-protoporphyrin O-methyltransferase (EC: 2.1.1.11), which catalyzes the transformation of $\mathrm{Mg}$ protoporphyrin IX to Mg-protoporphyrin IX 13-monomethyl ester, accompanied by a significant decrease in Mg-Proto IX. 

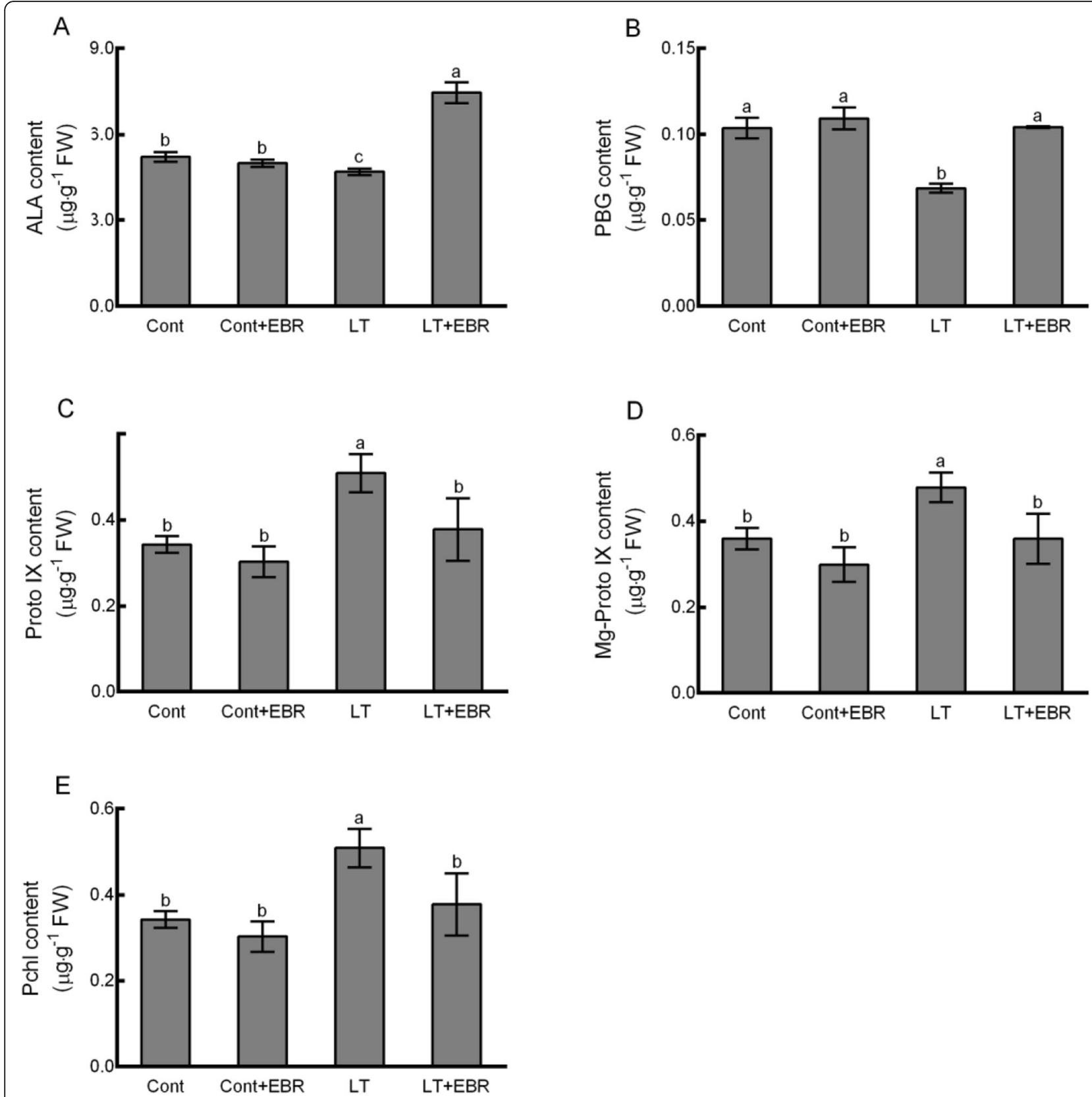

Fig. 8 Effects of exogenous EBR application on porphyrin and chlorophyll metabolism in wucai leaves under LT stress. Quantification of ALA content (a), PBG content (b), Proto IX content (c), Mg-Proto IX content (d) and Pchl content (e). The data are presented as the mean \pm SE. Bars with different letters above the columns indicate significant differences $(p<0.05$, Duncan's range test) on a given day of treatment

POR encodes a protochlorophyllide reductase (EC:1.3.1.33), which catalyzes the transformation of protochlorophyllide to chlorophyllide a, accompanied by a significant decrease in Pchl and a significant increase in photosynthetic pigments. These results suggest that applications of exogenous EBR can modulate the synthesis of endogenous ALA and its transformation to intermediate products, thereby improving chlorophyll biosynthesis.
Exogenous EBR weakened stress-induced photoinhibition and enhanced photosynthesis

Photosynthesis is among the primary plant processes that are affected most frequently by abiotic stress [35, 36]. PSII is believed to play an important role in plant responses to environmental stress [37]. For example, chilling stress has been shown to inhibit leaf photosynthesis through PSII-related photoinhibition [38, 39]. 


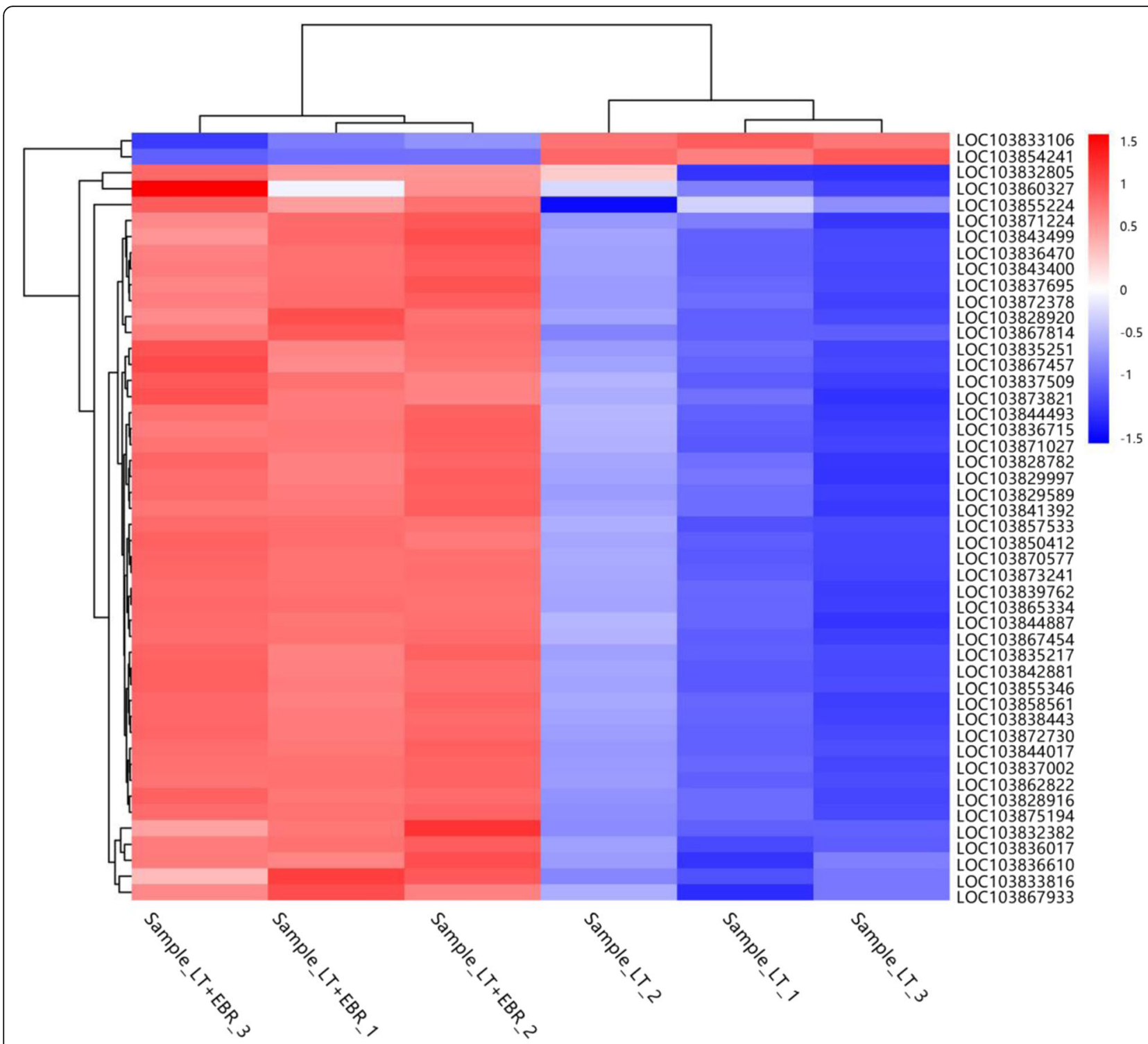

Fig. 9 Heat map of the photosynthesis and photosynthesis-antenna proteins-related DEGs in the LT and LT + EBR treatment of wucai leaves. The $\log _{2}$ FoldChange (FPKM) values were used to generate the heat maps using MeV software. Red and blue represent high or low expression levels, respectively, than those shown in white

Chlorophyll fluorescence transients quantitatively explain dynamic changes in the OJIP curve, which in turn quantitatively explains the PSII light energy absorption, conversion, electron transport, PSII action center, activity on the receptor and donor sides, and redox state of the electron transporter. In this study, GO and KEGG enrichment analyses suggest that EBR-mediated induction of photosynthesis, chloroplast thylakoid membrane, and chlorophyll biosynthesis may be the primary factors contributing to the distinct photosynthetic characteristics of the EBR transcriptome under LT conditions.

In contrast, the PSII activity level, which is reflected by $\phi_{\mathrm{Po}}$, was not affected in plants sprayed with EBR under normal temperature conditions (Fig. 7). $\phi_{\text {Po }}$ decreased in both LT and LT + EBR plants, especially in LT. This suggests that exogenous EBR can protect PSII under LT stress. Changes in $\mathrm{F}_{0}$ depend on the factors present during energy dissipation and PSII inactivation [40]. PSII damage or inactivation can result in an increase in $F_{0}$ [41]. In this study, $F_{0}$ increased under LT. However, the increase was smaller in LT + EBR than in LT (Fig. 7), indicating there was less relative damage to PSII in LT + EBR. Thus, exogenous EBR could alleviate damage caused by LT to photosynthetic reaction centers and reduce chilling photoinhibition. 
A
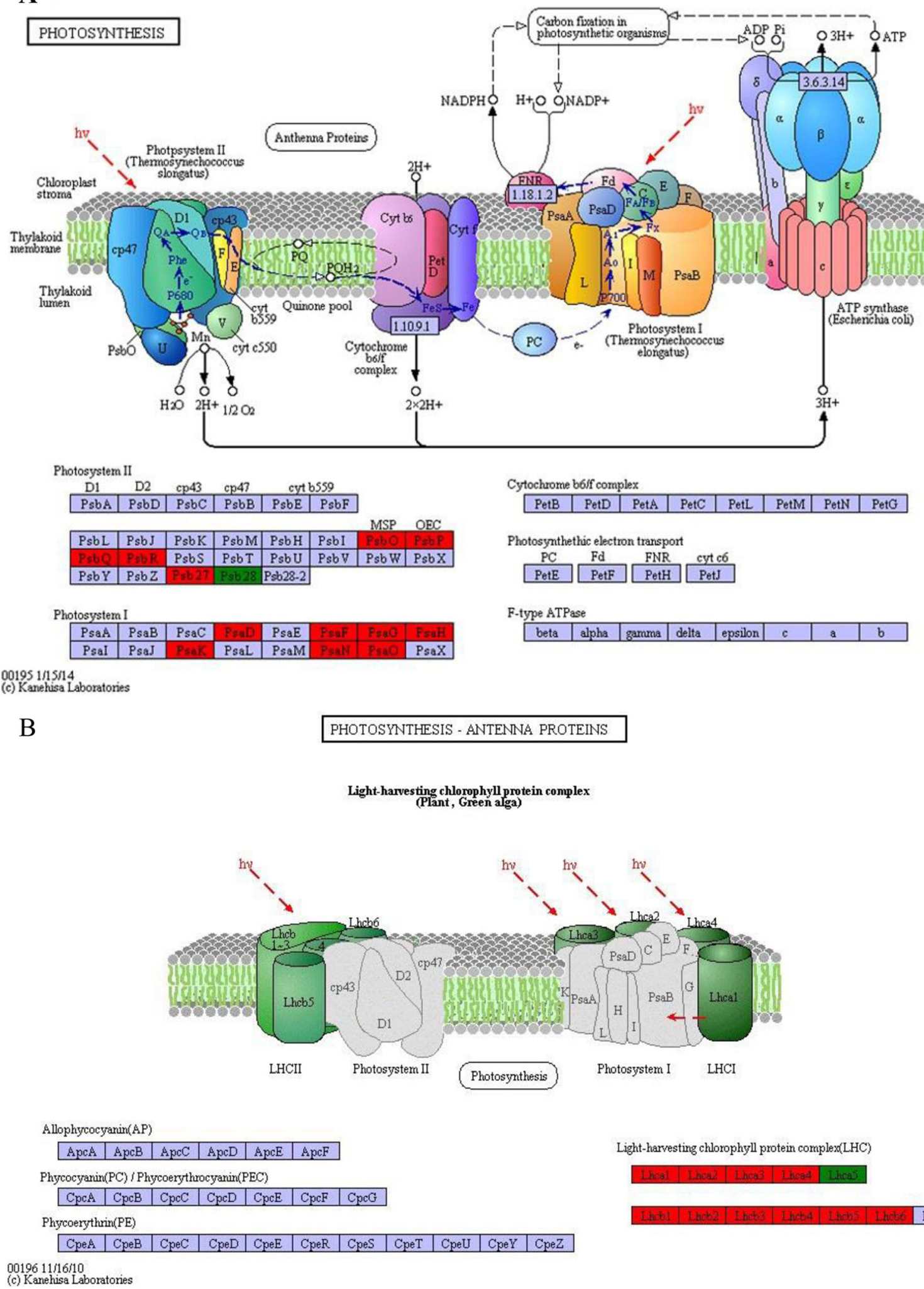

Fig. 10 Photosynthesis-related (A) and photosynthesis-antenna proteins-related (B) gene expression in wucai leaves influenced by LT alone or in combination with exogenous EBR application based on the KEGG pathway analysis. Red, green, and blue represent the up-, down-, and nonregulation of genes 

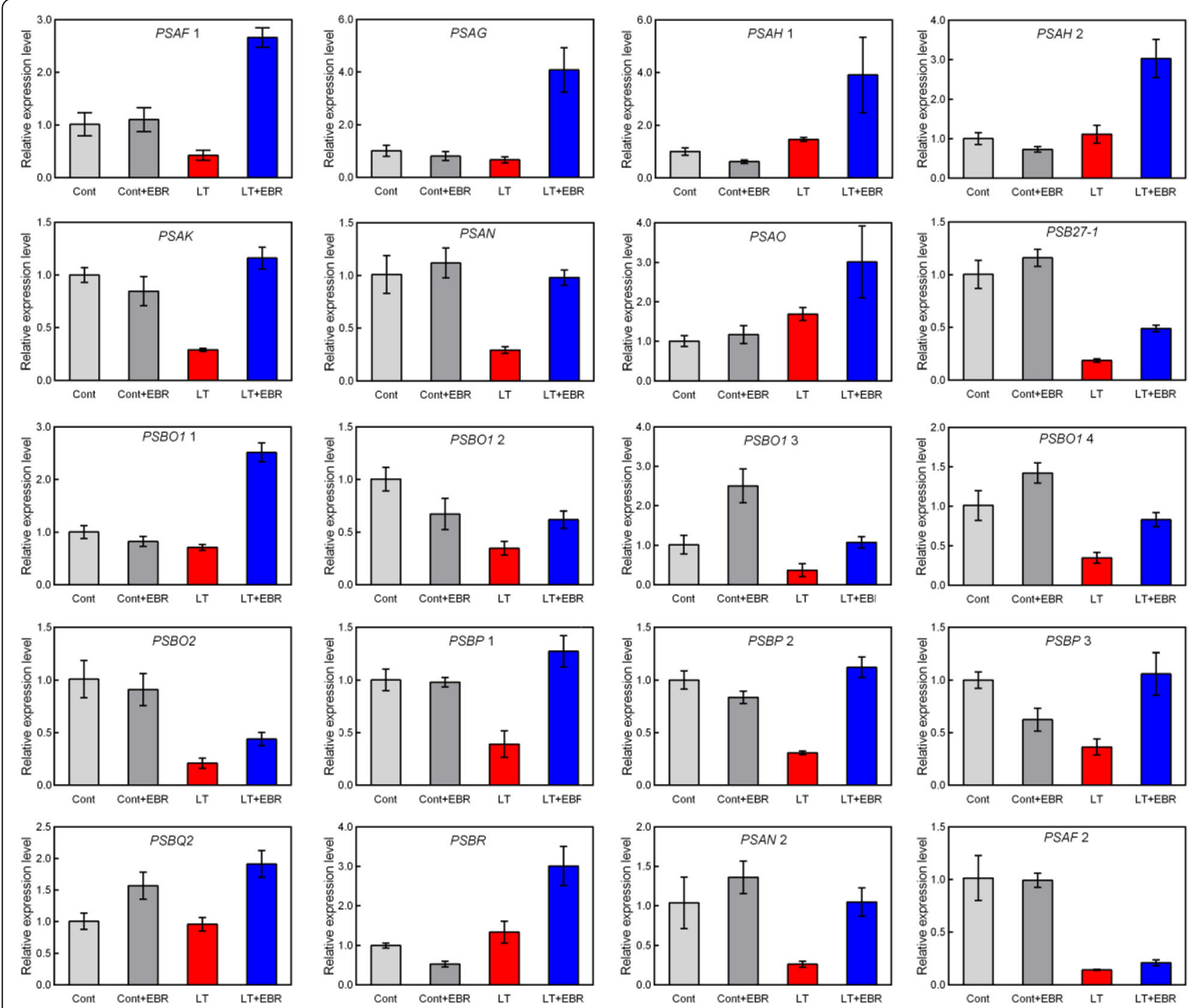

Fig. 11 Effects of exogenous EBR application on photosynthesis-related genes in wucai leaves under LT stress

The health of PSII is often used to determine the photochemical efficiency of the light reactions in photosynthesis II [42]. Compared to LT plants, the exogenous application of EBR in wucai promoted the maintenance of photochemical health under LT stress due to higher DPSII activity. From the KEGG pathway analysis, EBR application significantly regulated a series of proteins involved in PSII, including the oxygen-evolving enhancer, $10 \mathrm{kDa}$ and Psb27 proteins. The direct effect of EBR on these PSII proteins revealed that EBR could bind to PSII membranes in order to maintain their integrity and improve photosynthetic function despite osmotic stress [17].

\section{Conclusion}

This study provides insight into the function of exogenous EBR application on gene expression and predicted functions in wucai responding to LT stress. Compared to LT,
SLA and MSI were significantly improved in LT + EBR. Additionally, the content of MDA and total soluble sugar significantly decreased in LT +EBR, indicating that exogenous EBR could alleviate LT injury. The transcriptome analysis revealed that EBR primarily affected the transcripts associated with photosynthesis, porphyrin and chlorophyll metabolism under LT stress. Specifically, a significant increase in chlorophyll content and significant changes in the chlorophyll fluorescence parameters (i.e., $\mathrm{V}_{\mathrm{j}}, \mathrm{Fv} / \mathrm{F}_{0}$, and $\mathrm{PI}_{\mathrm{abs}}$ ) were detected when $\mathrm{LT}+\mathrm{EBR}$ was compared to LT. The regulation of the transcriptome in combination with the physiology of chlorophyll biosynthesis and photosynthesis demonstrates the protective mechanism of EBR treatment in response to LT stress. The DEGs identified here could be further investigated as molecular markers, or used in future functional analyses investigating responses to LT stress and EBR application. 


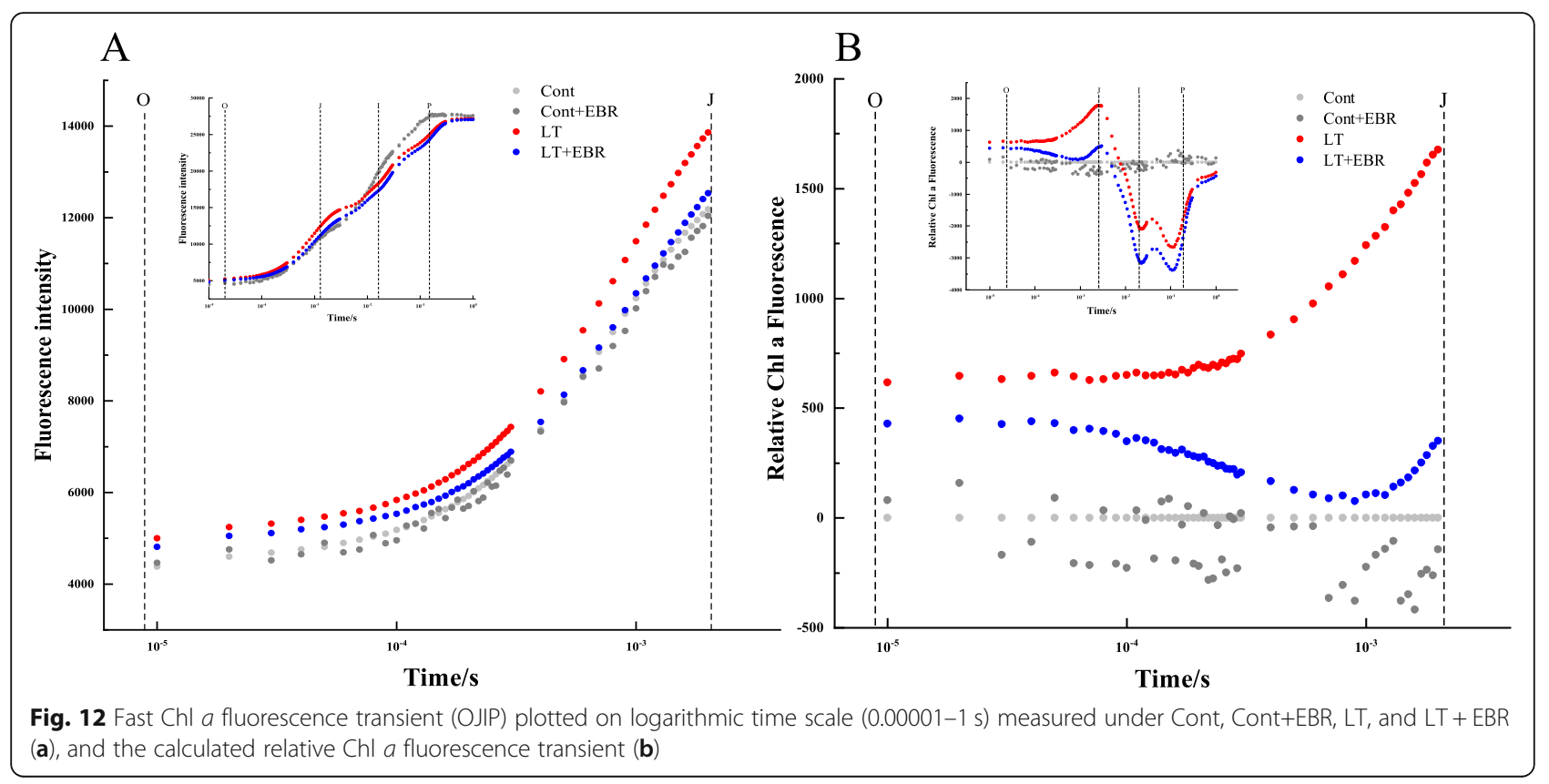

\section{Methods}

\section{Plant material and stress treatment}

Wucai seeds were provided by the Vegetable Genetics and Breeding Laboratory of the College of Horticulture, Anhui Agricultural University. Seeds were germinated from plugs before being transplanted into pots containing a mixture of stroma and vermiculite $(3: 1, v / v)$. Seedlings were grown in a growth chamber at $25 / 18{ }^{\circ} \mathrm{C}$ (day/ night), $300 \mu \mathrm{mol} \mathrm{m} \mathrm{m}^{-2} \mathrm{~s}^{-1}$ photon flux density, and $70 \%$ relative humidity with a 12:12 $\mathrm{h}$ photoperiod.

In a pilot study, 6 levels of EBR concentrations (0, 0.05, $0.08,0.1,0.12$, and $0.15 \mu \mathrm{M})$ were explored for treating wucai plants following a previously described protocol
[21]. Based on the results of the pilot study, $0.1 \mu \mathrm{M}$ EBR was identified as the optimum EBR concentration, which was subsequently used as the processing condition in the main experiment (Additional file 4: Figure S4).

Seedlings of uniform size at the 5-6 leaf stage (45 dafter planting) were selected and randomly divided into 2 groups for pretreatment. One group $(n=50)$ was sprayed with a $0.1 \mu \mathrm{M}$ EBR solution, while the other group ( $n=$ 50) was sprayed with the same volume of $d_{d d H_{2}} \mathrm{O}$. Two $d$ after pretreatment spraying, the seedlings in each pretreatment group were further divided into 2 groups. One group $(n=25)$ continued to grow in the suitable environment without LT stress, while the other group $(n=25)$
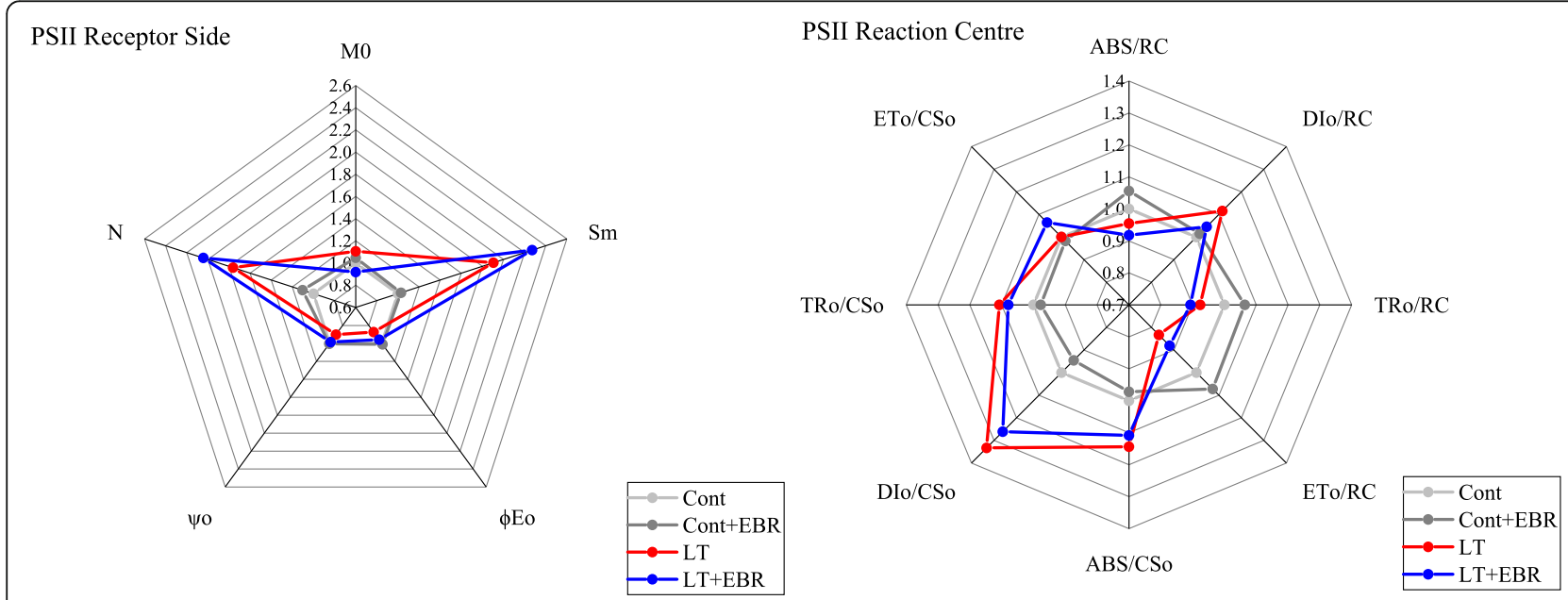

Fig. 13 Effects of exogenous EBR application on PSII in wucai leaves under LT stress 
$\mathrm{A}^{6000}$

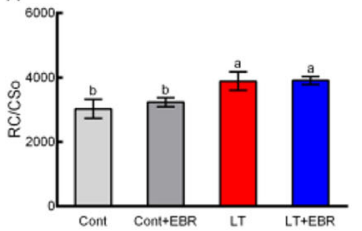

E
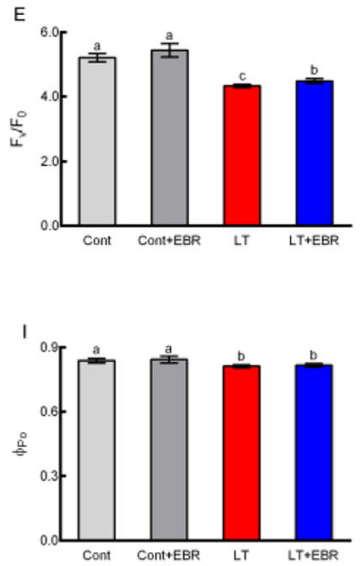
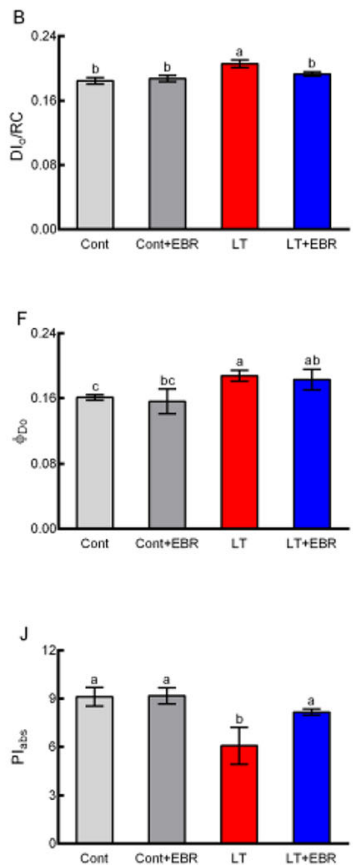

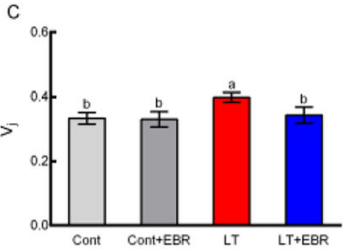

G

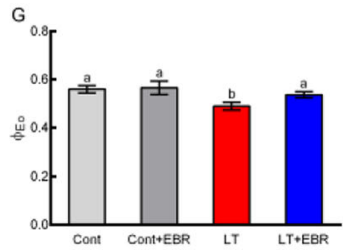

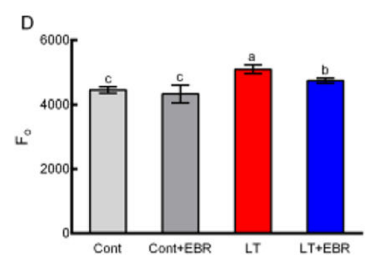

H

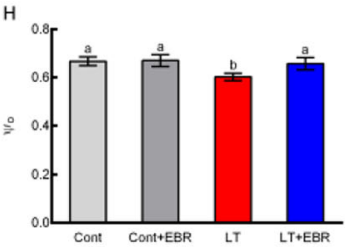

Fig. 14 Effects of exogenous EBR application on chlorophyll fluorescence parameters in wucai leaves under $L T$ stress. $A-J$ represent $R C / C S_{0}, D_{0} /$ $R C, V_{j}, F_{0}, F v / F_{0}, \varphi D o, \varphi E o, \psi_{0}, \varphi P o$ and $P_{a b s}$ respectively

was grown under LT. In total, there were 4 treatment groups: (1) Cont plants grown in an ideal environment with the $\mathrm{ddH}_{2} \mathrm{O}$ pretreatment; (2) Cont+EBR plants grown in an ideal environment with the $0.1 \mu \mathrm{M}$ EBR pretreatment; (3) LT plants grown with the $\mathrm{ddH}_{2} \mathrm{O}$ pretreatment; and (4) LT + EBR plants grown with the $0.1 \mu \mathrm{M}$ EBR pretreatment. Seedlings in both the LT and LT + EBR groups were transferred to a phytotron set at $7 / 3{ }^{\circ} \mathrm{C}$ (day/ night), $300 \mu \mathrm{mol} \mathrm{m}^{-2} \mathrm{~s}^{-1}$ photon flux density, and $70 \%$ relative humidity with a $12: 12 \mathrm{~h}$ photoperiod. Each treatment was replicated 3 times in the phytotron, and each treatment consisted of 25 plants. After $5 \mathrm{~d}$ of LT, functional leaf samples from 3 biological replicates were collected from LT and LT + EBR for sequencing. All of the samples were collected at the same time, ground into powder in liquid nitrogen, and immediately stored at $80^{\circ} \mathrm{C}$ for future analyses.

\section{Measurement the contents of SLA, MSI, Total soluble sugar and MDA}

SLA was determined according to Choong et al. [43] with minor modifications. SLA was calculated by dividing the area of 10 diameter leaf discs $(0.6 \mathrm{~mm})$. Then, the leaf samples were placed in a constant temperature drying oven set at $105^{\circ} \mathrm{C}$ for $15 \mathrm{~min}$, and subsequently dried at $75^{\circ} \mathrm{C}$ to a constant dry weight. SLA was calculated as the ratio of the leaf area to dry leaf weight.

MSI was determined according to methods previously described by Souhir et al. [44] with minor modifications.
Briefly, the same quantity of leaves was placed in a plugged test tube containing $10 \mathrm{~mL} \mathrm{ddH}_{2} \mathrm{O}$. Then the tube was placed in a $40{ }^{\circ} \mathrm{C}$ water bath for $30 \mathrm{~min}$. Afterwards, the conductivity was measured $(\mathrm{C} 1)$. Then, the tube was transferred to a $100{ }^{\circ} \mathrm{C}$ water bath for $10 \mathrm{~min}$, cooled to room temperature, and the conductivity was measured again (C2). MSI was calculated using the following formula:

$$
\text { MSI }=[1-(\mathrm{C} 1 \div \mathrm{C} 2) \times 100 \%]
$$

After grinding, dry leaf samples were mixed with $4 \mathrm{~mL}$ $80 \%$ alcohol and shaken at $80{ }^{\circ} \mathrm{C}$ for $30 \mathrm{~min}$. Cooled samples were centrifuged at $6855 \mathrm{r} / \mathrm{min}$ for $3 \mathrm{~min}$, and the supernatant was collected. The residue was washed with $80 \%$ alcohol 3 times, and the supernatant was extracted after each wash. Then, all of the supernatants were brought to a set volume of $10 \mathrm{~mL}$ with $80 \%$ alcohol and decolorized with $0.01 \mathrm{~g}$ activated carbon at $80{ }^{\circ} \mathrm{C}$ for $30 \mathrm{~min}$. After the addition of activated carbon, the material was filtered to obtain a filtrate, which was mixed with $6 \mathrm{~mL}$ anthrone and heated in a boiling water bath for $10 \mathrm{~min}$. Samples were then ice-cooled and kept at room temperature $\left(25^{\circ} \mathrm{C}\right)$ for $10 \mathrm{~min}$. After the chromogenic reaction, the optical density (OD) was spectrophotometrically recorded at $620 \mathrm{~nm}$.

MDA is a product of lipid peroxidation, associated with plant oxidative stress. The MDA level of all of the plant samples was assessed through lipid peroxidation 
following the methods previously described by Mohammadi et al. [45] with minor modifications. Fresh leaf samples $(0.5 \mathrm{~g})$ were homogenized in $10 \mathrm{~mL}$ trichloroacetic acid $(10 \%)$ and centrifuged at $4000 \mathrm{r} / \mathrm{min}$ for $10 \mathrm{~min}$. Then, $2.0 \mathrm{~mL}$ supernatant was mixed with $2 \mathrm{~mL}$ thiobarbituric acid $(0.6 \%)$, heated at $100{ }^{\circ} \mathrm{C}$ for $15 \mathrm{~min}$, and cooled to room temperature. The absorbance of each aliquot was measured at $450\left(\mathrm{~A}_{450}\right), 532\left(\mathrm{~A}_{532}\right)$, and $600 \mathrm{~nm}\left(\mathrm{~A}_{600}\right)$. The MDA content was calculated using the following formula:

$$
\mathrm{MDA}=6.45 \times\left(\mathrm{A}_{532}-\mathrm{A}_{600}\right)-0.56 \times \mathrm{A}_{450}
$$

\section{RNA extraction, library construction and RNA sequencing} Total RNA was extracted from the LT and LT + EBR leaf samples using a mirVana miRNA Isolation Kit (Ambion, TX, USA) following the manufacturer's instructions. RNA integrity was evaluated using an Agilent 2100 Bioanalyzer (Agilent Technologies, CA, USA). Samples with an RNA Integrity Number (RIN) $\geq 7$ were subjected to subsequent analysis. Libraries were constructed using the TruSeq Stranded mRNA LT Sample Prep Kit (Illumina, CA, USA) following the manufacturer's instructions. Then these libraries were sequenced on the HiSeqTM 2500 or HiSeq X Ten Illumina sequencing platform (Illumina, CA, USA), which generated $125 \mathrm{bp} /$ $150 \mathrm{bp}$ paired-end reads.

\section{Sequence assembly and annotation}

Each library generated $>6$ gigabytes of raw data. Clean reads were filtered from the raw sequencing data and low-quality reads containing unknown nucleotides or adaptor sequences were removed; this procedure was performed following the methods previously described by Bolger et al. [46]. Clean, filtered reads were aligned to the $B$. rapa reference genome by hisat2 [47].

\section{Differential expression analysis}

A differential expression analysis was performed for both LT and LT + EBR treatments based on the DESeq R package [48], which allowed for statistical analysis using a negative binomial (NB) distribution model. To control the false discovery rate (FDR), the FDR value calculation method was used to calculate the $p$-value of each gene. Then, the FDR error control method was used to perform multi-hypothesis test corrections on the $p$-value, where an adjusted $p$-value $<0.05$ and $\mid \log _{2}$ (foldchange) $\mid>1$ was accepted to represent DEGs.

GO enrichment analysis of the DEGs was performed according to the GOseq $\mathrm{R}$ package. GO terms with the number of DEGs $>2$ were regarded as significantly enriched $(p<0.05)$. Statistical enrichment of the DEGs was carried out in KEGG pathways using KOBAS software [49].

\section{Validation of DEGs by qRT-PCR}

Total RNA was extracted using the RNeasy extraction tool then reverse-transcribed into cDNA for the next analysis. A total of 20 transcripts were selected to verify the RNA-Seq analysis. Actin (ACT) was used as a reference gene to normalize the data. The SYBR fluorescent reagent (TaKaRa, RR820A) and Bio-Rad CFX96 ${ }^{\mathrm{Tm}}$ RealTime System (Bio-Rad, CA, USA) were used to conduct qRT-PCR following the manufacturer's instructions. The gene expression data were analyzed using the $2^{-\Delta \Delta C t}$ relative quantitative methods [50] in Microsoft Excel.

\section{Measurement of photosynthetic pigment content and chlorophyll biosynthesis intermediate contents}

Chlorophyll contents were determined using the methods previously described by Lichtenthaler et al. and Wellburn et al. [51] with minor modifications. Briefly, chlorophyll was extracted from $0.2 \mathrm{~g}$ leaves with $25 \mathrm{~mL}$ extracting solution (acetone/V: ethanol/V: water $/ \mathrm{V}=4.5$ : 4.5:1). The absorbance of the supernatant was measured at 3 wavelengths: 645, 652, and $663 \mathrm{~nm}$. The contents of Chl $a$, Chl $b$ and Total Chl were calculated using the following formulas:

$$
\begin{aligned}
& \mathrm{C}_{\text {Chla }}=13.95 \mathrm{~A}_{665}-6.88 \mathrm{~A}_{649}, \\
& \mathrm{C}_{\mathrm{Chlb}}=24.96 \mathrm{~A}_{649}-7.32 \mathrm{~A}_{665}, \\
& \text { Total Chl }=\mathrm{Chl} \mathrm{a}+\mathrm{Chl} \mathrm{b}
\end{aligned}
$$

In order to estimate the concentration of ALA, $0.5 \mathrm{~g}$ leaf samples were homogenized in $5 \mathrm{~mL}$ trichloroacetic acid (4\%) and the extract was centrifuged at 14,541 $\mathrm{r} /$ $\mathrm{min}$ for $15 \mathrm{~min}$. Then, $3 \mathrm{~mL}$ supernatant was added to $1.7 \mathrm{~mL}$ sodium acetate and $0.1 \mathrm{~mL}$ of acetylacetone. The mixture was placed in a boiling water bath for $10 \mathrm{~min}$ then cooled to room temperature. Two $\mathrm{mL}$ supernatant was thoroughly mixed with an Ehrlich- $\mathrm{Hg}$ reagent $(0.2 \mathrm{~g}$ $\mathrm{HgCl}_{2}+1 \mathrm{~g}$ P-dimethyl amino benzaldehyde $+42 \mathrm{~mL}$ glacial acetic acid $+8 \mathrm{~mL} 70 \%$ perchloric acid). After $15 \mathrm{~min}$ in darkness, the absorbance of the resulting solution was determined spectrophotometrically at $553 \mathrm{~nm}$ [52].

PBG content was measured following the methods previously described by Bogorad et al. [53]. Briefly, $0.5 \mathrm{~g}$ leaves were homogenized in $5 \mathrm{~mL}$ extraction buffer $(0.6 \mathrm{M}$ Tris and $0.1 \mathrm{M}$ EDTA, $\mathrm{pH}$ was adjusted to 8.2 with $\mathrm{HCl}$ ) and centrifuged for $10 \mathrm{~min}$ at $14,541 \mathrm{r} / \mathrm{min}$. Two $\mathrm{mL}$ supernatant was thoroughly mixed with $2 \mathrm{~mL}$ Ehrlich- $\mathrm{Hg}$ reagent. After incubating in darkness for $15 \mathrm{~min}$, the absorbance was measured at $553 \mathrm{~nm}$. 
The (Proto IX, Mg-Proto IX and Pchl contents were quantified following the methods previously described by Hodgins and Huystee et al. [54]. Briefly, leaf tissues were extracted with $80 \%$ alkaline acetone and centrifuged at $11,873 \mathrm{r} / \mathrm{min}$ for $10 \mathrm{~min}$ at $4{ }^{\circ} \mathrm{C}$. The absorbance of the supernatant was measured at 575, 590 and 628 nm. The contents of Proto IX, Mg-Proto IX and Pchl were calculated using the formula previously described by Hodgins et al. [54].

\section{Measurement of chlorophyll $a$ fluorescence transients}

In vivo chlorophyll a fluorescence emissions were measured in $30 \mathrm{~min}$ dark-adapted leaves with a continuous excitation fluorometer Pocket Plant Efficiency Analyzer (PEA, Hansatech, UK). Data were sampled at $10 \mu \mathrm{s}$ intervals for the first $300 \mu \mathrm{s}$, providing excellent time resolution of $F_{0}$ and the initial rise kinetics. The time resolution of digitization was switched to slower acquisition rates as the kinetics of the fluorescence signal slowed. Chlorophyll $a$ fluorescence transient was analyzed using the JIP-test formulae [55]. The fluorescence intensity at $20 \mu \mathrm{s}$ was considered to be $\mathrm{F}_{0}$, while the maximal fluorescence intensity was presumed to be equal to $F_{m}$, as the intensity was high enough to ensure the closure of all of the reaction centers (RCs) of PSII. Moreover, the fluorescence intensity at $300 \mu \mathrm{s}(\mathrm{F} 300 \mu \mathrm{s}), 2 \mathrm{~ms}$ (J-step, FJ), and 30 ms (I-step, FI) were also measured [56]. The following parameters refer to time 0 (the start of fluorescence induction): (a) the specific energy fluxes for absorption per reaction center $(\mathrm{ABS} / \mathrm{RC})$ ), trapping ( $\mathrm{TRo} /$ $\mathrm{RC})$, electron transport $(\mathrm{ETo} / \mathrm{RC})$, and dissipation at the level of the antenna chlorophylls (DIo/RC); and (b) the normalized total complementary area above the OJIP transient or total electron carriers per RC $\left(\mathrm{Sm}=\mathrm{EC}_{0} / \mathrm{RC}=\right.$ Area $\left./\left(\mathrm{Fm}-\mathrm{F}_{0}\right)\right)$. Performance index $(\mathrm{PI})$ on absorption basis was calculated as: PI (abs) $=(\mathrm{RC} /$ $\mathrm{ABS}) \cdot[\phi$ Po / (1- $\phi \mathrm{Po})][\psi \mathrm{o} /(1-\psi \mathrm{o})]$. Maximum quantum yield of primary photochemistry was calculated as: $\phi \mathrm{Po}=\mathrm{Fv} / \mathrm{Fm}=(\mathrm{Fm}-\mathrm{Fo}) / \mathrm{Fm}$ [57]. Other parameters measured in this study are provided (Additional file 12: Table S8).

\section{Statistical analyses}

All of the data from the 4 treatments were subjected to an analysis of variance (ANOVA). The mean separation was performed using the Fisher's protected least significant difference (LSD) test with a significance level of $p<$ 0.05. Analyses were conducted using SPSS v19.0 for Windows (SPSS Inc., USA). Figures were plotted using GraphPad Prism v7.0 and Origin Pro v9.1 software (OriginLab Corporation, MA, USA).

\section{Supplementary information}

Supplementary information accompanies this paper at https://doi.org/10. 1186/s12864-019-6191-2.

Additional file 1: Figure S1. The quality control of six samples. A-F represent $L T-1, L T-2, L T-3, L T+E B R-1, L T+E B R-2$, and $L T+E B R-3$, respectively.

Additional file 2: Figure S2. The reads mapping of six samples. A-F represent $L T-1, L T-2, L T-3, L T+E B R-1, L T+E B R-2$, and $L T+E B R-3$, respectively.

Additional file 3: Figure S3. The 20 DEGs we randomly selected for qRT-PCR assay in EBR-mediated LT stress.

Additional file 4: Figure S4. The results of identifying EBR concentration.

Additional file 5: Table S1. Summary of sequence assembly after illumine sequencing.

Additional file 6: Table S2. Number of reads sequenced and mapped to the Brassica rapa genome.

Additional file 7: Table S3. The expression patterns of the cold acclimation and cold-induced genes.

Additional file 8: Table S4. The expression patterns of the BRresponsive genes.

Additional file 9: Table S5. Primers of verifying genes.

Additional file 10: Table S6. Primers of photosynthesis genes. Additional file 11: Table S7. DEGs of photosynthesis-antenna proteins and photosynthesis.

Additional file 12: Table S8. Parameters derived from the OJIP transient for use in the current study.

\section{Abbreviations}

ALA: 5-aminolevulinic acid; B. rapa: Brassica rapa; BR: Brassinosteroids; Chl $a$ : Chlorophyll $a$; Chl b: Chlorophyll $b$; Cont: Control; Cont+EBR: Control with 24-epibrassinolide; DEG: Different expression gene; EBR: 24-epibrassinolide; GO: Gene Ontology; KEGG: Kyoto Encyclopedia of Genes and Genomes; LT + EBR: Low temperature with 24-epibrassinolide; LT: Low temperature; MDA: Malondialdehyde; Mg-proto IX: Mg-protoporphyrin IX; MSI: Membrane stability index; PBG: Porphobilinogen; Pchl: Protochlorophyllide; Proto IX: Protoporphyrin IX; PSI: Photosystem I; PSII: Photosystem II; qRT-PCR: Real time quantitative PCR; RPKMs: The reads per kilo bases per million reads; SLA: Specific leaf area; Total Chl: Total Chlorophyll

\section{Acknowledgements}

Not applicable.

\section{Authors' contributions}

$\mathrm{CW}$ is the project leader. $\mathrm{MZ}$ performed research and wrote the manuscript; $M Z$ and $L Y$ designed the experiments. $M Z$ and JW carried out the experiments. LY and SX helped to modify the manuscript. YZ, LN, GC, JH and SZ supervised the study. All authors have read and approved the final manuscript.

\section{Funding}

This work was supported by the National Key R \& D Program of China (2017YFD0101803) for the study and collection of data, the Major Science and Technology Projects of Anhui Province, China (17030701013) for analysis of data, the National Natural Science Foundation of China (No. 31701910) and the Natural Science Foundation of Anhui Province, China (1908085MC96) for interpretation of data.

Availability of data and materials

The raw RNA-Seq data used in this study have been deposited in the Nation Center for Biotechnology Information (NCBI) Sequence Read Archive (SRA) database under the accession number SRP200451 (https://www.ncbi.nlm.nih. gov/sra/SRP200451).

Ethics approval and consent to participate Not applicable. 


\section{Consent for publication}

Not applicable.

\section{Competing interests}

The authors declare that they have no competing interests.

\section{Author details}

${ }^{1}$ College of Horticulture, Vegetable Genetics and Breeding Laboratory, Anhui Agricultural University, 130 West Changjiang Road, Hefei 230036, Anhui, China. ${ }^{2}$ Provincial Engineering Laboratory for Horticultural Crop Breeding of Anhui, 130 West of Changjiang Road, Hefei 230036, Anhui, China. ${ }^{3}$ Wanjiang Vegetable Industrial Technology Institute, Maanshan 238200, Anhui, China.

\section{Received: 6 June 2019 Accepted: 15 October 2019} Published online: 06 November 2019

\section{References}

1. Xie S, Nie L, Zheng Y, Wang J, Zhao M, Zhu S, Hou J, Chen G, Wang C, Yuan L. Comparative proteomic analysis reveals that chlorophyll metabolism contributes to leaf color changes in Wucai ( Brassica campestris L.) responding to cold acclimation. J Proteome Res. 2019;18(6):2478-92.

2. Vardhini BV, Anjum NA. Brassinosteroids make plant life easier under abiotic stresses mainly by modulating major components of antioxidant defense system. Front Environ Sci. 2015;2.

3. Anwar A, Liu Y, Dong R, Bai L, Yu X, Li Y. The physiological and molecular mechanism of brassinosteroid in response to stress: a review. Biol Res. 2018; 51(1):46.

4. Siddiqui $\mathrm{H}$, Ahmed KBM, Hayat S. Comparative effect of 28homobrassinolide and 24-epibrassinolide on the performance of different components influencing the photosynthetic machinery in Brassica juncea L. Plant Physiol Biochem. 2018;129:198-212.

5. Siddiqui H, Hayat S, Bajguz A. Regulation of photosynthesis by brassinosteroids in plants. Acta Physiol Plant. 2018;40(3):59.

6. Choudhary SP, Yu JQ, Yamaguchi-Shinozaki K, Shinozaki K, Tran LS. Benefits of brassinosteroid crosstalk. Trends Plant Sci. 2012;17(10):594-605.

7. Yuan L, Shu S, Sun J, Guo S, Tezuka T. Effects of 24-epibrassinolide on the photosynthetic characteristics, antioxidant system, and chloroplast ultrastructure in Cucumis sativus L. under $\mathrm{Ca}\left(\mathrm{NO}_{3}\right)_{2}$ stress. Photosynth Res. 2012;112(3):205-14.

8. Yuan L, Wang J, Xie S, Zhao M, Nie L, Zheng Y, Zhu S, Hou J, Chen G, Wang C. Comparative Proteomics Indicates That Redox Homeostasis Is Involved in High- and Low-Temperature Stress Tolerance in a Novel Wucai (Brassica campestris L.) Genotype. Int J Mol Sci. 2019;20(15):3760

9. Li J, Yang P, Xie J, Jihua YU. Effects of 24- epibrassinolide on growth and antioxidant enzymes system in pepper roots under chilling stress. J Nucl Agric Sci. 2015;29(5):1001-8.

10. Huang Y, Zhao LQ, Kong QS, Cheng F. Niu ml, Xie JJ, Nawaz MA, Bie ZL. Comprehensive mineral nutrition analysis of watermelon grafted onto two different rootstocks. Horticultural Plant J. 2016;2(2):105-13.

11. Khan TA, Yusuf M, Ahmad A, Bashir Z, Saeed T, Fariduddin Q, Hayat S, Mock HP, Wu T. Proteomic and physiological assessment of stress sensitive and tolerant variety of tomato treated with brassinosteroids and hydrogen peroxide under low-temperature stress. Food Chem. 2019;289:500-11.

12. Wei LJ, Deng XG, Zhu T, Zheng T, Li PX, Wu JQ, Zhang DW, Lin HH. Ethylene is Involved in Brassinosteroids Induced Alternative Respiratory Pathway in Cucumber (Cucumis sativus L.) Seedlings Response to Abiotic Stress. Front Plant Sci. 2015;6:982.

13. Ma L, Coulter JA, Liu L, Zhao Y, Chang Y, Pu Y, Zeng X, Xu Y, Wu J, Fang Y, et al. Transcriptome Analysis Reveals Key Cold-Stress-Responsive Genes in Winter Rapeseed (Brassica rapa L.). Int J Mol Sci. 2019;20(5):1071.

14. Jaglo KR, Kleff S, Amundsen KL, Zhang X, Haake V, Zhang JZ, Deits T, Thomashow MF. Components of the Arabidopsis C-repeat/dehydrationresponsive element binding factor cold-response pathway are conserved in Brassica napus and other plant species. Plant Physiol. 2001;127(3):910-7.

15. Li J, Yang P, Gan YT, Yu JH, Xie JM. Brassinosteroid alleviates chillinginduced oxidative stress in pepper by enhancing antioxidation systems and maintenance of photosystem II. Acta Physiol Plant. 2015;37(11):222.

16. Eremina M, Unterholzner SJ, Rathnayake Al, Castellanos M, Khan M, Kugler KG, May ST, Mayer KF, Rozhon W, Poppenberger B. Brassinosteroids participate in the control of basal and acquired freezing tolerance of plants. Proc Natl Acad Sci U S A. 2016;113(40):E5982-91.
17. Ma Y, Shukla V, Merewitz EB. Transcriptome analysis of creeping bentgrass exposed to drought stress and polyamine treatment. PLoS One. 2017;12(4): e0175848.

18. Cheng F, Liu S, Wu J, Fang L, Sun S, Liu B, Li P, Hua W, Wang X. BRAD, the genetics and genomics database for Brassica plants. BMC Plant Biol. 2011; 11(1):136.

19. Mortazavi A, Williams BA, McCue K, Schaeffer L, Wold B. Mapping and quantifying mammalian transcriptomes by RNA-Seq. Nat Methods. 2008;5(7): 621-8.

20. Niu KJ, Ma HL. The positive effects of exogenous 5-aminolevulinic acid on the chlorophyll biosynthesis, photosystem and Calvin cycle of Kentucky bluegrass seedlings in response to osmotic stress. Environ Exp Bot. 2018; 155:260-71.

21. Li J, Yang P, Kang J, Gan Y, Yu J, Calderon-Urrea A, Lyu J, Zhang G, Feng Z, Xie J. Transcriptome analysis of pepper (Capsicum annuum) revealed a role of 24-Epibrassinolide in response to chilling. Front Plant Sci. 2016;7:1281.

22. Dobrikova AG, Vladkova RS, Rashkov GD, Todinova SJ, Krumova SB, Apostolova EL. Effects of exogenous 24-epibrassinolide on the photosynthetic membranes under non-stress conditions. Plant Physiol Biochem. 2014;80:75-82.

23. Janeczko A, Gruszka D, Pociecha E, Dziurka M, Filek M, Jurczyk B, Kalaji HM Kocurek M, Waligorski P. Physiological and biochemical characterisation of watered and drought-stressed barley mutants in the HvDWARF gene encoding C6-oxidase involved in brassinosteroid biosynthesis. Plant Physiol Biochem. 2016;99:126-41.

24. Shu S, Tang Y, Yuan Y, Sun J, Zhong M, Guo S. The role of 24epibrassinolide in the regulation of photosynthetic characteristics and nitrogen metabolism of tomato seedlings under a combined low temperature and weak light stress. Plant Physiol Biochem. 2016;107:344-53.

25. Pociecha E, Dziurka M, Waligorski P, Krepski T, Janeczko A. 24-Epibrassinolide pre-treatment modifies cold-induced photosynthetic acclimation mechanisms and Phytohormone response of perennial ryegrass in cultivardependent manner. J Plant Growth Regul. 2017;36(3):618-28.

26. Wang D, Heckathorn SA, Mainali K, Tripathee R. Timing effects of heat-stress on plant Ecophysiological characteristics and growth. Front Plant Sci. 2016;7: 1629.

27. Niinemets $U$, Kull O. Biomass investment in leaf lamina versus lamina support in relation to growth irradiance and leaf size in temperate deciduous trees. Tree Physiol. 1999;19(6):349-58.

28. Li W, Timin S, Bing X, Chen LS, Shuang S, Peng J, Yonghua Z. Glycine betaine reduces chilling injury in peach fruit by enhancing phenolic and sugar metabolisms. Food Chem. 2018;272:530-38.

29. Chakraborty N, Basak J. Exogenous application of methyl jasmonate induces defense response and develops tolerance against mungbean yellow mosaic India virus in Vigna mungo. Funct Plant Biol. 2018;46(1):69-81.

30. Katz JJ, Norris JR, Shipman LL, Thurnauer MC, Wasielewski MR. Chlorophyll function in the photosynthetic reaction center. Annu Rev Biophys Bioeng. 1978;7(1):393-434

31. Niu JH, Anjum SA, Wang R, Li JH, Liu MR, Song JX, Zohaib A, Lv J, Wang SG, Zong XF. Exogenous application of brassinolide can alter morphological and physiological traits of Leymus chinensis (Trin.) Tzvelev under room and high temperatures. Chil J Agr Res. 2016;76(1):27-33.

32. Wang R, Anjum SA, Niu JH, Liu MR, Li JH, Zohaib A, Song JX, Lv J, Wang SG, Zong XF. Exogenous application of Brassinolide ameliorate chilling stress in Leymus Chinensis (Trin.) Tzvel. By modulating morphological, physiological and biochemical traits. Bangl J Bot. 2016;45(1):143-50.

33. Yang AJ, Anjum SA, Wang L, Song JX, Zong XF, Lv J, Zohaib A, Ali I, Yan R, Zhang Y. Effect of foliar application of brassinolide on photosynthesis and chlorophyll fluorescence traits of Leymus chinensis under varying levels of shade. Photosynthetica. 2017:1-11.

34. Turan S, Tripathy BC. Salt-stress induced modulation of chlorophyll biosynthesis during de-etiolation of rice seedlings. Physiol Plant. 2015;153(3): 477-91.

35. Allen DJ, Ort DR. Impacts of chilling temperatures on photosynthesis in warm-climate plants. Trends Plant Sci. 2001;6(1):36-42.

36. Chaves MM, Flexas J, Pinheiro C. Photosynthesis under drought and salt stress: regulation mechanisms from whole plant to cell. Ann Bot. 2009; 103(4):551-60.

37. Sui N, Han GL. Salt-induced photoinhibition of PSII is alleviated in halophyte Thellungiella halophila by increases of unsaturated fatty acids in membrane lipids. Acta Physiol Plant. 2014;36(4):983-92. 
38. Aro EM, McCaffery S, Anderson JM. Photoinhibition and D1 protein degradation in peas acclimated to different growth irradiances. Plant Physiol. 1993;103(3):835-43.

39. Zhang Z, Jia Y, Gao H, Zhang L, Li H, Meng Q. Characterization of PSI recovery after chilling-induced photoinhibition in cucumber (Cucumis sativus L.) leaves. Planta. 2011;234(5):883-9.

40. Guo YY, Tian SS, Liu SS, Wang WQ, Sui N. Energy dissipation and antioxidant enzyme system protect photosystem II of sweet sorghum under drought stress. Photosynthetica. 2018;56(3):861-72.

41. Meng C, Sui N. Overexpression of maize MYB-IF35 increases chilling tolerance in Arabidopsis. Plant Physiol Biochem. 2019;135:167-73.

42. Cornic G, Fresneau C. Photosynthetic carbon reduction and carbon oxidation cycles are the main electron sinks for photosystem II activity during a mild drought. Ann Bot. 2002;89(7):887-94.

43. Choong TW, He J, Qin L, Lee SK. Quality of supplementary LED lighting effects on growth and photosynthesis of two different Lactuca recombinant inbred lines (RILs) grown in a tropical greenhouse. Photosynthetica. 2018;56(4):1278-86.

44. Abdelkrim S, Jebara SH, Jebara M. Antioxidant systems responses and the compatible solutes as contributing factors to lead accumulation and tolerance in Lathyrus sativus inoculated by plant growth promoting rhizobacteria. Ecotoxicol Environ Saf. 2018;166:427-36.

45. Mohammadi M, Modarres-Sanavy SAM, Pirdashti H, Zand B, TahmasebiSarvestani Z. Arbuscular mycorrhizae alleviate water deficit stress and improve antioxidant response, more than nitrogen fixing bacteria or chemical fertilizer in the evening primrose. Rhizosphere. 2019:9:76-89.

46. Bolger AM, Lohse $\mathrm{M}$, Usadel B. Trimmomatic: a flexible trimmer for Illumina sequence data. Bioinformatics. 2014;30(15):2114-20.

47. Daehwan K, Ben L, Salzberg SL. HISAT: a fast spliced aligner with low memory requirements. Nat Methods. 2015;12(4):357-60.

48. Anders, S. and Huber, W. Differential expression of RNA-Seq data at the gene level - the DESeq package. Embl. 2013.

49. Chen X, Xizeng M, Jiaju H, Yang D, Jianmin W, Shan D, Lei K, Ge G, Chuan-Yun L, Liping W. KOBAS 2.0: a web server for annotation and identification of enriched pathways and diseases. Nucleic Acids Res. 2011:39(suppl):W316-22.

50. Livak KJ, Schmittgen TD. Analysis of relative gene expression data using real-time quantitative PCR and the 2(-Delta Delta $C(T))$ method. Methods. 2001;25(4):402-8

51. Lichtenthaler HK, Wellburn AR. Determinations of total carotenoids and chlorophylls $a$ and $b$ of leaf extracts in different solvents. Biochem Soc Trans. 1983;11(5):591-2.

52. Beaven GH. Biochemical spectroscopy. FEBS Lett. 1975 .

53. Bogorad L. Porphyrin synthesis. Methods Enzymol. 1962;5:885-95.

54. Hodgins RR, Van Huystee RB. Rapid simultaneous estimation of protoporphyrin and mg-porphyrins in higher plants. J Plant Physiol. 1986;125(3):311-23.

55. He L, Yu L, Li B, Du N, Guo S. The effect of exogenous calcium on cucumber fruit quality, photosynthesis, chlorophyll fluorescence, and fast chlorophyll fluorescence during the fruiting period under hypoxic stress. BMC Plant Biol. 2018;18(1):180.

56. Han $\mathrm{S}$, Tang $\mathrm{N}$, Jiang $\mathrm{HX}$, Yang LT, Li Y, Chen LS. CO $\mathrm{CO}_{2}$ assimilation, photosystem II photochemistry, carbohydrate metabolism and antioxidant system of citrus leaves in response to boron stress. Plant Sci. 2009;176(1):143-53.

57. Zribi L, Fatma G, Fatma R, Salwa R, Hassan N, Néjib RM. Application of chlorophyll fluorescence for the diagnosis of salt stress in tomato 'Solanum lycopersicum (variety Rio Grande)'. Sci Hortic. 2009;120(3):367-72.

\section{Publisher's Note}

Springer Nature remains neutral with regard to jurisdictional claims in published maps and institutional affiliations.

Ready to submit your research? Choose BMC and benefit from:

- fast, convenient online submission

- thorough peer review by experienced researchers in your field

- rapid publication on acceptance

- support for research data, including large and complex data types

- gold Open Access which fosters wider collaboration and increased citations

- maximum visibility for your research: over $100 \mathrm{M}$ website views per year

At $\mathrm{BMC}$, research is always in progress.

Learn more biomedcentral.com/submissions 